\title{
A THEORETICAL AND EXPERIMENTAL STUDY OF PLASMA-WAVE COUPLING AND PROPAGATION USING PHASED WAVEGUIDE ARRAYS AND ELECTROSTATIC STRUCTURES
}

BY

S, BERNABEI, M, A. HEALD, W. M. HOOKE, R, W. MOTLEY, $F, J$, PAOLONI AND

M. BRAMBILLA AND W. D, GETTY

\section{PLASMA PHYSICS LABORATORY}

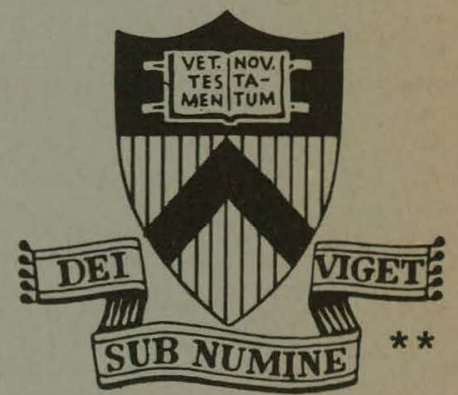

\section{PRINCETON UNIVERSITY PRINCETON, NEW JERSEY}

This work was supported by U. S. Energy Research and Development Administration Contract E(11-1)-3073. Reproduction, translation, publication, use and disposal, in whole or in part, by or for the United States Government is permitted. 


\section{DISCLAIMER}

This report was prepared as an account of work sponsored by an agency of the United States Government. Neither the United States Government nor any agency Thereof, nor any of their employees, makes any warranty, express or implied, or assumes any legal liability or responsibility for the accuracy, completeness, or usefulness of any information, apparatus, product, or process disclosed, or represents that its use would not infringe privately owned rights. Reference herein to any specific commercial product, process, or service by trade name, trademark, manufacturer, or otherwise does not necessarily constitute or imply its endorsement, recommendation, or favoring by the United States Government or any agency thereof. The views and opinions of authors expressed herein do not necessarily state or reflect those of the United States Government or any agency thereof. 


\section{DISCLAIMER}

Portions of this document may be illegible in electronic image products. Images are produced from the best available original document. 
This report was prepared as an account of work sponsored by the United States Government. Neither the United States nor the United States Energy Research and Development Administration, nor any of their employees, nor any of their contractors, subcontractors, or their employees, makes any warranty, express or implied, or assumes any legal liability or responsibility for the accuracy, completeness or usefulness of any information, apparatus, product or process disclosed, or represents that its use would not infringe privately owned rights.

Printed in the United States of America.

Available from

National Technical Information Service

U, S. Department of Commerce 5285 Port Royal Road Springfield, Virginia 22151

Price: Printed Copy \$_; Microfiche $\$ 1.45$

*Pages

$$
\begin{gathered}
1-50 \\
51-150 \\
151=325 \\
326-500 \\
501=1000
\end{gathered}
$$

NTIS

Selling Price

$\$ 4.00$

5.45

7.60

10.60

13.60 
A Theoretical and Experimental Study of Plasma-Wave Coupling and Propagation Using Phased Waveguide Arrays and Electrostatic Structures

S. Bernabei, M. A. Heald*, W. M. Hooke

R. W. Motley, F. J. Paoloni

Princeton University, Plasma Physics Laboratory

Princeton, New Jersey 08540, USA

M. Brambilla

C.E.N.-G Grenoble, France

W. D. Getty

Electron Physics Laboratory, University of Michigan

Ann Arbor, Michigan USA

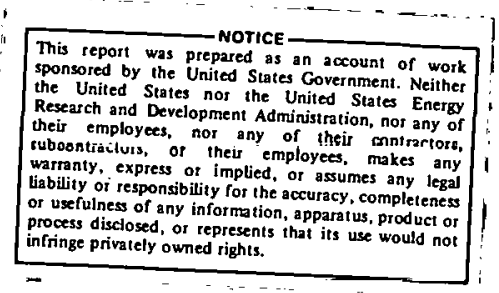


A THEORETICAL AND EXPERIMENTAL ŚTUDY OF

PLASMA-WAVE COUPLING AND PROPAGATION USING

PHASED WAVEGUIDE ARRAYS AND ELECTROSTATIC STRUCTURES

S. BERNABEI, M. A. HEALD*, W. M. HOOKE,

R. W. MOTLEY, F. J. PAOLONI

Plasma Physics Laboratory, Princeton University

Princeton, New Jersey 08540 U.S.A.

M. BRAMBILLA

C.E.N.-G. Grenoble, France

and

W. D. GETTY

Electron Physics Laboratory, University of Michigan Ann Arbor, Michigan U.S.A.

\begin{abstract}
A theoretical and experimental study of the coupling and propagation of electron plasma waves excited by waveguide arrays and electrostatic structures is presented. The waves are excited in a low temperature, linear plasma column in a homogeneous magnetic field. Inder appropriate conditions efficient coupling to plasma waves can be obtained. These studies are of relevance to plasma heating in Lhal lie modes are identical to those that must be generated in any lower hybrid heating experiment.
\end{abstract}

\footnotetext{
*Permanent address: Department of Physics, Swathmore college, Swathmore, Pennsylvania 19081.
} 


\section{INTRODUCTION}

A frequently proposed approach to the problem of plasma heating is the generation and subsequent damping of large amplitude waves near the lower hybrid resonance frequency. For large tokamaks and proposed reactors this characteristic frequency is of the order of a few gigahertz, and in this range microwave power sources exist which are capable of delivering continuous wave power of up to one megawatt. The output of microwave tubes is easily combined at these frequencies so that multi-megawatt, continuous wave, power sources could be constructed. Since the free space wavelength is in the decimeter range, it has been emphasized by a number of authors that open-ended waveguide structures are an especially simple technique for the introduction of supplementary heating power. A particularly attractive system consists of a phased array of waveguides which has been studied in detail theoretical, y by Brambilla:[1]. In this paper we give the results of theorctical and experimental studies of phased array coupling. In addition, we show the mode structure and the penetration of the waves in the linear and nonlinear regimes.

These experiments were performed on a linear device known as $\mathrm{H}-1$. We emphasize that the frequencies in this experimental study are well above the maximum lower hybrid frequency in:the $\mathrm{H}-1$ plasma. However, both theory and experiment show that the coupling is completely determined by the relatively tenuous plasma facing the waveguide array where the wave frequency is near the electron plasma 
frequency, and thus the results are of consequence for tokamak plasmas.

\section{WAVE EXCITATION AND PLASMA PENETRATION}

As a guide to the design and discussion of an efficient coupling structure, it is useful to summarize a few qualitative properties of wave propagation in the frequency range of. interest [For detailed discussion see Appendix A]. An important constraint on the antenna design comes from the existence of a cut-off at a critical electron density, $n_{c^{\prime}}$, where the local plasma

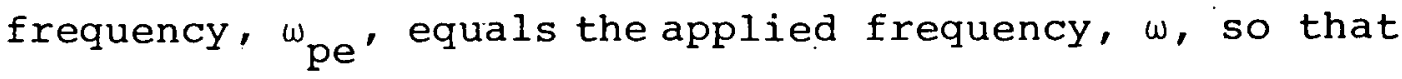

$$
\mathrm{n}_{\mathrm{c}}=\frac{\omega^{2} \mathrm{~m}_{\mathrm{e}}}{4 \pi \mathrm{e}^{2}}
$$

If $\omega$ is chosen to approximate the lower hybrid frequency at the plasma center (peak density $n_{p}$ ) under conditions such that $\omega_{L H} \approx \omega_{p i}$.' then $\mathrm{n}_{\mathrm{c}} \approx \mathrm{m}_{\mathrm{e}} / \mathrm{m}_{\mathrm{i}} \mathrm{n}_{\mathrm{p}}$ and the critical layer is close to the plasma edge. waves with $\mathrm{n}_{n}{ }^{2}=\mathrm{k}_{n}{ }^{2} \mathrm{c}^{2} / \mathrm{\omega}^{2}<1$ (where $\mathrm{k}_{n}$ is the wavevector component along the static magnetic field) become evanescent beyond the critical layer and are therefore totally reflected there. "Slow" waves with $k_{n}{ }^{2} \mathrm{c}^{2} / \omega^{2}>1$, however, after tunneling across the evanescence region between the antenna and the critical layer, progagate freely towards higher densities.

It is well known $[2,3,4]$ that the lower hybrid resonance is accessible from outside the plasma only for waves which satisfy the more stringent condition

$$
\mathrm{n}_{n}{ }^{2}>1+\left(\omega_{\mathrm{pe}}^{2} / \omega_{\mathrm{ce}}^{2}\right)_{\text {res }}
$$


where the electron plasma and cyclotron frequencies are evaluated at the hybrid resonance layer. For a localized antenna, the group velocity propagates along the "resonant cones" [5, 6], the trajectory becoming asymptotic to the lower hybrid layer. Waves

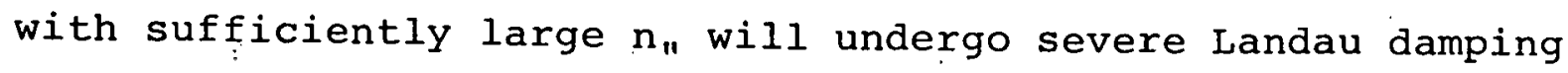
as they propagate into the hot plasma interior [7]. Thus, we expect effective penetration when the spectral distribution of the antenna field has a well defined peak around a $k_{n}$ value satisfying the condition $\omega / v_{e}>k_{\|}>\omega / c$ where $v_{e}$ is the electron thermal velocity. 'l'he coupling device, theretore, must be a slow-wave structure which cannot radiate into a vacuum, but should couple to freely propagating plasma waves.

In the intermediate range,

$$
1<n_{1}{ }^{2}<1+\left(\omega_{p e}{ }^{2} / \omega_{c e}{ }^{2}\right)_{\text {res }},
$$

the waves suffer reflection before reaching the resonance layer. Most of this reflected energy will not couple back into the antenna, but will travel along the plasma surface away from the coupler. A slow wave structure which couples efficiently to waves in this intermediate region may be useful tor moditying the surface layers of a plasma, but should be avoided if one wishes to heat the interior. The phased arrays in these experiments are designed so that the bulk of their associated power spectrum satisfies condition (2).

In order to achicve cfficient coupling, nne must. impose the correct electric and magnetic field polarization while simultaneously satisfying the above conditions on the $k_{n}$ spectrum. 
As discussed in appendix $A$, at densities just above the critical density, the electric field of the lower hybrid or "slow" mode is mainly parallel to the magnetic field. Well above this critical density the electric field becomes nearly parallel to the density gradient and longitudinal in the sense that $\overline{\mathrm{E}}$ and $\overline{\mathrm{k}}$ are parallel. These considerations suggest that for moderate density gradients the best choice for the excitation electric field is along the static magnetic field. If the gradient is very steep, however, there might be some advantage to imposing an electric field parallel to the density gradient. Although no calculations for this latter case are presently available, we have obtained some empirical results to justify this conclusion.

In either case, the required configuration can be provided by a waveguide mounted perpendicular to the vacuum chamber. Electric fields parallel to the static magnetic fields can be obtained by a retangular waveguide excited in the $\mathrm{TE}_{10}$ mode. Electric fields perpendicular to the static magnetic fields can be provided by waveguides excited in a TM mode. To achieve accessibility and yet maintain propagation within the guide, the TM waveguide must be dielectrically loaded - a requirement not necessary for $\mathrm{TE}_{10}$ excitation.

For a single $\mathrm{TE}_{10}$ guide the excited spectrum will be peaked at $\mathrm{n}_{n}=0$ and the fraction of energy in the $\mathrm{n}_{n}{ }^{2}>1$ portion of the power spectrum will be an inverse function of the small side dimension of the guide [4]. A fundamental improvement in the control over the power spectrum can be obtained by using a properly phased array of waveguides $[1,8,9]$. The study of these phased $\mathrm{TE}_{10}$ arrays constitutes the central theme of this paper. 


\section{THEORY}

For the reasons discussed above, a phased $\mathrm{TE}_{10}\left(\mathrm{E}_{\mathrm{n}} \mathrm{B}\right.$ ) array or "grill" of waveguides should produce a more effective coupler than: a single guide. For the grill, the first peak in the $n_{\text {" }}$ spectrum is given roughly by $n_{n}=c N \Delta \phi / \omega L$ where $\mathrm{N}$ is the number of waveguides, $\Delta \phi$ the phase difference between adjacent guides, and $L$ the total width of the array. It is important to note that one may steer the radiation parallel or anti-parallel to the static magnetic field by appropriately adjusting $\Delta \phi$. Thus in a tokamak, for example, one might choose the phase so that the waves propagate parallel to the Ohmic heating current and will not interact with runaway electrons. Alternatively, one may choose a sufficiently high $n_{\text {" }}$ so that the wave will undergo strong Landau damping.

In computing the coupling of the grill to the plasma, one must calculate the self-consistent waveguide-plasma electromagnetic fields rather than the vacuum field of the coupler alone. Mutual coupling between the waveguides and excitation of higher evanescent modes at the waveguide throats cause the excited spectrum to depart significantly from that associated with the vacuum fields. To obtain quantitative results, calculations have been performed using an idealized two-dimensional model as sketched in Fig. 1. The waveguides are assumed to be infinite in the vertical direction; the plasma chamber occupies the $x \geq 0$ halfspace with the plasma edge at $x=x_{p} \geq 0$. The density gradient is along $x$ and the static magnetic field along $z$. 
The field in the waveguides can be written as a superposition of eigenmodes [1],

$$
\begin{aligned}
E_{z}= & \sum_{p=1}^{N} \theta_{p}(z) \sum_{n=0}^{v}\left[\alpha_{n p} \exp \left(i k_{n} x\right)+\beta_{n p} \exp -\left(i k_{n} x\right)\right] \cos \frac{n \pi\left(z-z_{p}\right)}{b} \\
& +\sum_{n=v+1}^{\infty} \beta_{n p} \exp \left(\gamma_{n} x\right) \cos \frac{n \pi\left(z-z_{p}\right)}{b}
\end{aligned}
$$

and similar expressions for $\mathrm{E}_{\mathrm{x}}$ and $\mathrm{B}_{\mathrm{y}}$ (the other components vanish by symmetry). Here

$$
\begin{aligned}
& k_{n}=\left(\varepsilon \omega^{2} / c^{2}-n^{2} \pi^{2} / b^{2}\right)^{1 / 2} \text { for propagating modes, } \\
& \gamma_{n}=\left(n^{2} \pi^{2} / b^{2}-\varepsilon \omega^{2} / c^{2}\right)^{1 / 2} \text { for evanescent modes; }
\end{aligned}
$$

where $b$ is the width of the waveguides, $\varepsilon_{r}$ the relative dielectric constant of the material in the guides, ${ }_{. p}(z)$ is a bookkeeping function which determines the amplitude and phase of the electric field in front of the $p$-th waveguide $\left(z_{p}<z<z_{p}+b\right)$ and is zero elsewhere, and $\alpha_{\mathrm{pn}}$ is the complex amplitude of the $\mathrm{nth}$ excited mode in the p-th guide. The reflection coefficients and the coefficients of excitation of evanescent modes, $\beta_{n p}$ have to be evaluated.

The electromagnetic field for $x \geq 0$ is similarly written as a Fourier integral over $k_{\|}$; for example: 


$$
E_{z}=\int \sigma\left(k_{11}\right)\left[\exp \left(i k_{\perp} x\right)-Y\left(k_{n}\right) \exp -\left(i k_{\perp} x\right)\right] \exp \left(\ddot{i} k_{1 \prime} z\right) d k_{\| \prime} \text {, }
$$

where $k_{\perp}^{2}=\left(\omega^{2} / c^{2}-k_{\|}^{2}\right)$ in vacuum, and the integration path must run above the $k_{\|}=+\omega / c$ branch point and below the $k_{n}=-\omega / c$ branch point. $Y\left(k_{11}\right)$ then describes the wave reflected back from the plasma for each $k_{\|}$. According to the above discussion, $Y\left(k_{1}\right)$ is mainly determined by the relatively thin low density layer below and around the critical density. In this region the "slow" plasma wave is fairly accurately described (to terms of order $\left.m_{e} / m_{i}\right)$ by the equation

$$
\frac{d^{2} E}{d x^{2}}+\frac{\omega^{2}}{c^{2}} \cdot\left(1-n_{n}{ }^{2}\right) \quad P E_{z}=0
$$

where $\mathrm{P}$ is the $\mathrm{zz}$ component of the cold plasma dielectric tensor. The exact solution of this equation around the regular turning point $P=0$ must be matched to the appropriate wKB solution at sufficiently high densities. This can be done analytically for a number of functional dependences of $P$ upon $x$ [4]. In all the calculations shown in this work, a linear density profile was assumed with zero denisity at the waveguide edge. In thïs case

$$
Y\left(k_{\|}\right)=\frac{1-Z}{1+z} \quad Z=\frac{J_{1 / 3}(\zeta)+J_{-1 / 3}(\zeta)}{J_{2 / 3}(\zeta)-J_{-2 / 3}(\zeta)}
$$


for $\mathrm{k}_{n}{ }^{2} \mathrm{c}^{2} / \omega^{2}<1$, and

$$
Y\left(k_{n}\right)=\frac{1-Z^{\prime}}{1+Z^{\prime}} \quad Z^{\prime}=\frac{I_{1 / 3}(\zeta)+\exp (-\pi i / 3) I_{-1 / 3}(\zeta)}{I_{-2 / 3}(\zeta)+\exp (-\pi i / 3) I_{2 / 3}(\zeta)},
$$

for $\mathrm{k}_{n}{ }^{2} \mathrm{c}^{2} / \omega^{2}>1$, where the dimensionless quantity $\zeta$, is given by

$$
\zeta=2.83 \cdot 10^{11}\left|1-\mathrm{n}_{n}{ }^{2}\right|^{1 / 2} \frac{\omega^{3}}{\mathrm{c}^{3}}\left(\frac{\mathrm{dn}}{\mathrm{dx}}\right)_{\mathrm{x}=0}^{-1}
$$

in cgs units.

Imposing the continuity of $\vec{E}$ and $\vec{H}$ at the waveguide throats, and the vanishing of $\mathrm{E}_{z}$ at the metallic wall of the chamber, one obtains the following linear system for the unknown coefficients $\beta_{n p}:$

$$
\beta_{m q}+\sum_{p=1}^{N} \sum_{n=1}^{\infty} \kappa_{m n}(q, p) \dot{\beta}_{n p}=\alpha_{m q}-\sum_{p=1}^{N} \sum_{n=1}^{v} \kappa_{m n}(q, p) \alpha_{n p} \text {, }
$$

where

$$
k_{m, n}(q, p)=C_{m} \int d k_{n} \frac{F_{m q}^{*}\left(k_{n}\right) F_{n p}\left(k_{n}\right)}{k_{\perp}} \frac{I+Y\left(k_{n}\right)}{1-Y\left(k_{n}\right)},
$$

with

$$
\begin{aligned}
& F_{n p}\left(k_{1 \prime}\right)=-\frac{i k_{n}}{2 \pi} \frac{1-(-1)^{n} \exp \left(-i k_{n} b\right)}{k n^{2}-n^{2} \pi^{2} / b^{2}} \\
& C_{0}=-\frac{2 \pi \omega}{c b} . \\
& C_{m}=-\frac{4 \pi}{b} k_{m} \text {. for } 1 \leqslant m \leqslant v
\end{aligned}
$$




$$
c_{m}=\frac{4 \pi i}{b} \gamma_{m} \quad \text { for } m \geq v+1
$$

This system, truncated to include up to 10 eigenmodes, is solved numerically to obtain the coefficients $\beta_{n p}$ for various conditions of waveguide phase and density gradient. 


\section{PLASMA SOURCE AND DIAGNOSTICS}

The plasma source, shown in Fig. 2, is a modified version of the radio frequency source described by Motley, et al., [10]. The machine consists of a $23 \mathrm{~cm}$ diameter stainless-steel vacuum vessel $270 \mathrm{~cm}$ long, which is immersed in a uniform $( \pm 0.58)$ dc magnetic field whose strength can be varied up to $16 \mathrm{kG}$. The plasma excitation system consists of a copper cylindex $2.0 \mathrm{~cm}$ long which is wrapped around an alumina supporting cylinder of internal diameter $9.5 \mathrm{~cm}$. The space between the vacuum vessel and the alumina is sealed at each end with an aluminum ring and is evacuated to prevent arcing between the copper cylinder and the main vacuum vessel. Up to $7 \mathrm{~kW}$ of $\mathrm{rf}$ power at $155 \mathrm{MHz}$ is supplied to the copper cylinder in $400 \mu \mathrm{sec}$ pulses. The repetition rate is 10 pulses per second and data in a given time interval is stored between pulses using

a Boxcar Integrator unit. A smaller cylindrical electrode, suspended coaxially at one:end: of the machine and driven continuously by 10 watts of power at $45 \mathrm{MHz}$, is used to maintain a weak steady-state discharge between high-power pulses.

The line-integrated electron density is measured with an $8 \mathrm{~mm}$. microwave interferometer and the density profile is derived from a movable Langmuir probe biased at $-90 \mathrm{~V}$ to collect ion saturation current. Electron density gradients near the plasma edge are estimated from this probe current profile, which is assumed to be proportional to the electron density. The peak density of an argon plasma can be varied up to about $6 \times 10^{12} \mathrm{~cm}^{-3}$, and density gradients of up to about $2 \times 10^{13} \mathrm{~cm}^{-4}$ are 
obtained. Under our conditions; the ion and electron temperatures are uniform across most of the plasma radius and are $2 \mathrm{eV}$ at the end of the plasma senerating pulse, at which time the antenna systems are energized.

The wave propagation region is bounded by a stainlesssteel liner of internal diameter $11.5 \mathrm{~cm}$. The purpose of the liner is to restrict the plasma diameter to approximateiy that of the alumina cylinder and to provide a well-defined boundary to the plasma column. The triaxial electric probe, which is movable in both axial and radial directions, is used to sample the wave signal in the plasma as well as to measure the Langmuir density profile. The wave amplitude is observed with a square-law video detector and the phase is obtained by mixing the probe signal with a fixed reference signal and recording the resulting interference as the probe is moved.

\section{ANIENNA SYSTEMS}

In order to investigate the efficiency of coupling and penetration of slow waves into the plasma, we have constructed several types of launching structures which operate at $3.4 \mathrm{GHz}$. This frequency was chosen such that the free-space wavelength is of the order of the diameter of the plasma column, a scaling relation typical of lower hybrid heating experiments in present day tokamak devices. The $\mathrm{TE}_{10}$ group of antennas are constructed of standard WR229 (2.290 x $1.145 \mathrm{in.)}$ waveguide, the open mouths of which are machined in an arc to match the curvature of the conducting liner. A photograph of the single, double, and quadruple waveguide antenna systems is shown in Fig. 3. The TE 
systems are divided as follows:

1. Single $\mathrm{TE}_{10}$ waveguide.

A single WR229. waveguide operating in the $\mathrm{TE}_{10}$ mode and oriented such that either the electric field is parallel to the dc magnetic field (the "ordinary" polarization) or perpendicular to the magnetic field. ("extraordinary") .

2. Double $\mathrm{TE}_{10}$ waveguide.

A WR229 waveguide divided by a central partition parallel to the broad wall and mounted such that the waveguide can be moved with respect to the liner. The two guides are ariven in the TE 10 mode with relative phase controlled by a calibrated phase shifter. The spectrum has a peak $n_{\text {" }}$ value that can be varied from zero up to 2.2. For high power work $(2 \mathrm{~kW})$, a teflon filled double waveguide of dimensions 2.29" x 1.5", driven at $2.45 \mathrm{GHz}$ was used.

3. Quadruple $\mathrm{TE}_{10}$ waveguide.

A. WR229 waveguide equally divided by three partitions parallel to the broad wall. The four guides are driven in the $\mathrm{TE}_{10}$ mode with relative phases controlled by the circuit shown in Fig. 4. By selecting the relative phases of the wave-

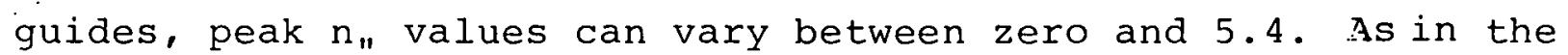
double waveguide, the energy can be directed upstream or downs.tream of the plasma by selecting $\pm 90^{\circ}$ phase shifts between adjacent guides.

In order to generate electric fields parallel to the density gradient, TM ancl electrosicatic coupling systems have also been constructed. These systems, shown in Fig. 5, are:

1. : Circular $\mathrm{TM}_{11}$, dielectric-loaded waveguide. 
A circular loaded waveguide of diameter $4.7 \mathrm{~cm}$ constructed using a low-loss epoxy medium of dielectric constant 9.5. The dielectric is shielded from the plasma by a thin ceramic plug of dielectric constant 9.4. The coax-waveguide coupling stubs are driven out of phase by an external circuit using a hybrid junction and the corresponding aperture spectrum has a peak corresponding to $\mathrm{n}_{11}=2.2$.

2. Balanced shielded pair transmission line.

A $100 \Omega$ shielded-pair transmission line which is open-ended and has an outer diameter of $4.4 \mathrm{~cm}$. The inner conductors, which are driven out of phase, can be projected (or retracted) up to $3 \mathrm{~cm}$ beyond the outer conductor. The inner conductors are aligned in the same plane as the magnetic field and provide an $\mathrm{n}_{i}$ spectrum which peaks at 2.2 . 


\section{RESULTS AND DISCUSSION}

\subsection{SINGLE WAVEGUIDE}

Under certain conditions a single waveguide may act as an efficient antenna for launching slow plasma waves. If, for example, the narrow dimension of the waveguide is reduced so that a substantial fraction of the-wave energy at the waveguide throat is concentrated in slow waves $\left(n_{n}>1\right)$, and if the plasma is overdense $\left(n_{e}>n_{c}\right)$ within a fraction of a wavelength of the throat, then most of the microwave energy may be converted into plasma waves. In Fig. 6 we show nominal wavelength spectra of waveguides whose short dimensions vary from 0.33 to 0.07 of a wavelength. It is readily seen that a substantial fraction of the power propagates with $n_{!}>1$ and can therefore penetrate an overdense plasma.

We have obtained experimental results on the coupling of single waveguides to an overdense plasma.. In Fig. 7 we show the power reflected from a single WR2 29 waveguide excited in the $\mathrm{TE}$ ol mode $\left(\mathrm{E}_{\mathrm{O}} \mathrm{B}_{\mathrm{O}}\right)$ as a function of the average density gradient in front of the guide. Typical shot-to-shot variation in the reflected power is about $10 \%$. Both theory and experiment show that the reflected power is quite insensitive to the density gradient, but the the experimental points scatter about $45 \%$ reflection while the theory predicts $30-40 \%$ reflection. One can also measure the reflectivity for wave excitation with $E_{\perp} B_{0}$. These data show an increase in refiection from $30 \%$ to $85 \%$ as the density gradient is changed from $10^{12}$ to $10^{1.3} \mathrm{~cm}^{-4}$.

In a related set of measurements we have determined the 
power reflection as a function of the waveguide dimensions. The data" of Fig. 8 show that coupling to the plasma improves as the small dimension of the waveguide is reduced to $0.1 \lambda$. The theory predicts a reflection minimum around $b \approx 7 \mathrm{~mm}$, but the minimum was not observed experimentally. Such a minimum was observed if the density gradient were reduced below $10^{12} \mathrm{~cm}^{-4}$, but the increase in reflection was not as pronounced as theory would predict. It should be emphasized that close agreement should not be expected for coupling where the waveguide size is a small fraction of a wavelength, since the counling in this regime is a sensitive function of both the boundary conditions and the position and exact shape of the density profile.

It is clear from the discussion above that close coupling between a single thin waveguide and plasma can be achieved. We believe, however, that waveguide arrays are to be preferred in practical applications to plasma heating, both to broaden the spatial extent of the wave packet launched and to increase the power handling capacity of the waveguide.

\subsection{DOUंBLE WAVEGUIDE}

The behaviour of the double waveguide radialing into a vacuum can be simply described in terms of partial waves that are reflected at each aperture, cross-coupled to the neighboring guide, or radiated. As discussed in Appendix B, the two return waves in each guide interfere destructively when $\Delta \phi=0$ producing a total reflection minimum, and constructively for 
$\Delta \phi=180^{\circ}$ producing a total reflection maximum : The antenna cannot radiate efficiently in.this latter case since $n_{\text {" }}$ is greater than unity while the total refractive index in the vacuum must remain equal to unity.

Some experimental results for the variation of total reflection coefficient with phase areshown in Fig. 9. Also included are calculations from the theory described in the previous section which uses the experimentally determined linear density gradient near the plasma edge closest to the antenna. The measured and computed.figures for the vacuum situation show close agreement while those for the plasma have good qualitative but only fair quantitative agreement. This is not surprising considering the simplifying assumptions used in the theoretical model.

Computations shown in Fig. 10 indicate that an optimum in the coupling when $\Delta \phi=180^{\circ}$. can be achieved when the density gradient is about $4 \times 10^{12} \mathrm{~cm}^{-4}$. The experimental results suggest a minimum at a somewhat higher gradient but both theoretical and experimental results indicate a very broad minimum. We have also probea the interior of the plasma column to ascertain the characteristics of the waves excited. In Fig. 11 we illustrate the spreading of the wave packet inside the overciense plasma column. 
The leading edge of the wave packet follows the resonance cone trajectory, corresponding to $n_{n}>1$. In some cases the wave packet became distinctly bimodal, one component remaining at a fixed radius just inside the $\omega_{\text {pe }}=\omega$ layer. Interferometric measurements show $n_{n} \sim 1.3$ near the outer edge of the plasma, and $n_{n} \sim 3$ near the leading edge of the penetrating wave packet [11]. Quantitative ray-tracing calculations, derived from the full electromagnetic dispersion relation, are given in Fig. 12 . These show that the portion of spectral energy with lower values of $n_{\text {" }}$ is essentially trapped in the electron density layer between $\mathrm{n}_{\mathrm{C}}$ and $\mathrm{n}_{\mathrm{l}}$ ' (the point of maximum penetration for a given $n_{n}$ - see Appendix A),

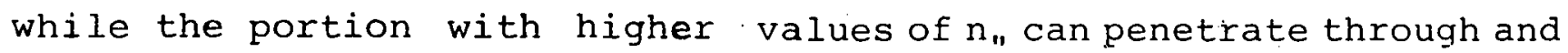
beyond our maximum densities. For certain antenna conditions and density profiles, these calculations are consistent with the observed separation into a trapped and a penetrating component.

By withdrawing the antenna behind the conducting liner, we can artifically increase the thickness of the evanescent layer, and the reflection coefficient rises accordingly. Similarly, when the antenra is projected beyond the liner wall, forming its own plasma limiter, we achieve higher density gradients than are otherwise available. It is difficult to estimate the values of gradient so obtalned, but there is evidence, consistent with the model calculations, that at sufficiently high gradients the reflection coefficient of the double waveguide increases somewhat. It is also possible that at high densities there may be a significant plasma concentration inside the waveguides, thereby increasing the reflection within each guide. 


\subsection{QUADRUPLE WAVEGUIDE ARRAY}

An array of four adjacent waveguides possesses certain distinct advantages over either a single or a double waveguide system. First of all, the greater number of elements provides a better defined parallel wavelength spectrum. Secondly; the quadruple array allows redistribution of the wave energy into any value of parallel refractive index between 0 and $\sim \lambda / 2 b$. Thirdly, one has the option of directing the beam in either direction from the array by phasing adjacent elements to be approximately $90^{\circ}$ out of phase. Each of these possibilities is illustrated in the nominal power spectra of Fig. 13.

Experimental data on the reflection characteristics of individual elements and the total reflection from a quadruple waveguide array are compared with the theory in Figs. 14 and 15. Both vacuum and plasma loaded waveguides exhibit reflection characteristics in qualitative agreement with theory. The best coupling (at this particular density gradient) occurs at $\Delta \phi=60^{\circ}$, corresponding to $\mathrm{n}_{\|} \approx 1.8$. The directional characteristics of the antenna are evident from the signals on the rf probe, which clearly show a $5 \mathrm{~dB}$ asymmetry between upstream and downstream power.

The influence of density gradient on the total reflected power from a four-waveguide array is shown in Fig. 16 for a phase difference of $60^{\circ}$. Again, a broad minimum around $\nabla n=2 \times 10^{12} \mathrm{~cm}^{-4}$ is predicted and observed, with the experimental points somewhat above the theoretical line. Comparable reflection coefficients 
$(\sim 20 \%)$ are observed when the phase configuration of the four waveguides is simulating the double waveguide. Both theory and experiment show the minimum reflection for the quadruple waveguide to be somewhat inferior to that for the double waveguide with respect to low reflection coefficient over a range of density gradients. This is probably caused by the finite size of the partition dividing the waveguides.

Calculations have been made for the quadruple wavequide array in which the input power is divided such that the amplitude of the inner waveguides is reduced relative to that of the outer. Optimum coupling is predicted when the inner waveguide signals are $9 \mathrm{~dB}$ below the outer for our typical parameters, a situation not far different from excitation of the outer quides onlv. While theoretically this latter case differs from the thickwall double waveguide, in that a ground plane (the wall) is replaced by parasitic waveguides, experimentally we find the two cases indistinguishable.

\subsection{CIRCULAR TM TII $_{11}$ DIECTRIC-LOADED WAVEGUIDE}

A loaded $\mathrm{TM}_{11}$ waveguide provides a large electric field component parallel to the density gradient. The nodes of the waveguide fields are in the plane parallel to the magnetic field and, for our antenna, the parallel refractive index is approximately 2.2 .

Some data for the power reflection coefficient versus density gradient is given in Fig. 17. Coupling improves as the density gradient increases since the width of the critical layer decreases and coupling to the $\mathrm{E}_{\mathbf{x}}$ component of the slow wave is possible. At 
the highest gradient the power reflection was less than $40 \%$. Probe measurements within the plasma showed wave propagation to the expected depth of penetration for the given $n_{\text {" }}$ and density profile. For the highest gradient the depth of penetration was about one centimeter. We speculate that the coupling and wave penetration would continue to improve at even higher gradients provided the $n_{\|}$was sufficiently large to ensure wave penetration. This type of antenna, and perhaps dielectric-loaded antennas in general, may be attractive in situations with very steep gradients.

\subsection{ELECTROSTATIC COUPLERS}

Wavelaunchers consisting of open-ended coaxial and shieldedpair transmission lines possess certain similarities to electrostatic plate launchers, used in many recent experiments $[6,12,13]$, in that they allow one to launch waves with high values of $n_{n}$. When the inner conductors are withdrawn inside the outer, these systems will preferentially excite the TMol and $\mathrm{TM}_{11}$ circular, waveguide modes, respectively, which are evanescent and do not radiate efficiently into either a vacuum or a plasma. When the inner conductors are projected beyond the outer conductor and the surrounding ground plane, the configuration begins to look like a dipole antenna. Figs. 18 and 19 present data for the shielded pair as a function of the projection of the inner conductors and of density gradient, respectively. We observe significant coupling and wave penetration only when the inner conductors are projected into the plasma. 


\subsection{HIGH POWER EXCITA'TION}

The coupling theory discussed previously is completely linear, i.e., the incident wave is assumed not to affect the plasma column, and the electrostatic wave excited inside the plasma is assumed to be stable. However, if the amplitude of the plasma wave becomes extremely large, a nonlinear interaction between the wave and the plasma column may occur. The parametric decay, for example, of lower hybrid waves into other lower hybrid waves and sound waves has been predicted theoretically $[14,15]$ and observed experimentally [16, 17]. In addition. to this interaction, there has been the recent suggestion [18] that the lower hybrid waves will undergo self focussing in the outer layers of plasma and may not be able to penetrate to the interior. Our main consideration in this work is to establish whether these or other nonlinear interactions. prevent the wave from propagating through the surtace layers of the plasma.

Recent theory and experiment [14, 15] have demonstrated that an intense high frequency wave may decay. parametrically into two daughter waves, the most common decay involving transformation to a low-frequency ion wave and a high-frequency electrostatic wave. Accuinjanying the wave dccay there is efficient ion or electron heating. Nonlinear interactions giving rise to anomalously high plasma heating rates would, in general be beneficial unless the interaction occurs in the low density outer edges of the plasma column. Accordingly, we have examined the nonlinear effects occuring in our plasma column where 
the linear coupling is well understood.

Parametric interaction does not occur in a plasma unless the high frequency pump wave supplies sufficient energy to overcome the damping of the two daughter waves. We limit ourselves to intermediate frequencies $\left(\omega_{\mathrm{LH}} \ll \omega_{0} \ll \omega_{\mathrm{pe}}\right)$, and assume that the waves are purely electrostatic, such that the dispersion. relation for the pump wave is $\omega_{0}=\omega_{\text {pe }} \cos \theta_{0}$, for the highfrequency daughter $\omega_{2}=\omega_{\text {pe }} \cos \theta_{2}$, and for the ion wave $\omega_{1}=k_{1}\left(\mathrm{KT}_{e} / \mathrm{m}_{i}\right)^{1 / 2}$. Furthermore we assume that. $\left|\mathrm{k}_{\mathrm{o}}\right| \sim\left|\mathrm{k}_{1}\right|=\left|\mathrm{k}_{2}\right|$. and çonsider that the ion wave is damped by electron Landau damping and the electron plasma wave by collisions. One can then simplify the threshold criterion [14] to

$\left(\frac{e E_{c}}{m \omega_{o} v_{e}}\right)^{2}=\sqrt{\frac{\pi}{2}\left(\frac{m_{e}}{m_{i}}\right)^{1 / 2}} \frac{1}{\cos \theta} \frac{v_{e o}}{\omega_{2}} \frac{\cos ^{2} \theta+\left(\omega_{o}^{2} / \omega_{c e}{ }^{2}\right) \sin ^{2} \theta}{\cos ^{4} \theta+\left(\omega_{o}^{2} / \omega_{c e}^{2}\right) \sin ^{4} \theta}$,

where $E_{c}$ is the critical electric field, $v_{e}=\left(2 \mathrm{KT}_{e} / \mathrm{m}_{e}\right)^{1 / 2}, v_{e o}$ is the electron-atom collision frequency, and 0 is the angle between the phase velocity of the pump wave and the magnetic field. With our plasma parameters $E_{C} \sim 6 \mathrm{~V} / \mathrm{cm}$. The equation is invalid at low frequencies $\left(\omega_{0} \approx 10^{6}\right)$, where ion-atom collisions are important, and at high frequencies $\left(\omega_{0} \tilde{>} 5 \times 10^{7}\right)$, where electron Landau damping of the electron plasma wave may not neglected.

The experimental arrangement to investigate nonlinear interactions was very similar to that described in section 4, with 
the exception that the signal frequency was lowered to $2.45 \mathrm{GHz}$, supplied by a pulsed (10-100 $\mu \mathrm{sec}) 2 \mathrm{~kW}$ magnetron. The split waveguide was filled with teflon $\left(\varepsilon_{r}=2.3\right)$ to lower the cut-off frequency well below $2.45 \mathrm{GHz}$. The power level should be adequate to produce nonlinear behavior, since. the maximum electric field (inside the waveguide) was $\sim 400 \mathrm{~V} / \mathrm{cm}$, well above the threshold field for the excitation of parametric instabilities.

The coupling efficiency between the waveguide and the plasmawas found to be almost independent of the input level. For a given phase shift between the two waveguides, the reflectivity was observed to change very little with input power up to a maximum of $2 \mathrm{~kW}$. When $\Delta \phi=180^{\circ}$ there is perhaps a slight improvement in the coupling at the higher power levels. These results are illustrated in Fig. 20 . The wave trajectories inside the plasma column were also measured. Again, as shown in Fig. 21, there is little change in the radial distribution of the wave amplitude as the electrostatic pump wave propagates down the plasma column. This result was obtained with an rf pulse width less than $20 \mathrm{\mu s}$. If the pulse width were extended to $\sim 100 \mu \mathrm{s}$ there was an apparent shift of $\sim 0.5$. cm outward. This shift was associated with a broadening of the plasma column by the additional ionization on the side of the plasma facing the waveguide and disappeared if the rf pulse were shortened to avoid changes in the plasma profile. No evidence for the nonlinear filamentation instability' was found. 
We have also examined the wave spectrum inside the plasma with the rf probe placed $5 \mathrm{~cm}$ from the waveguide at the radius of maximum wave potential. It was necessary to time resolve these measurements using a boxcar integrator to avoid frequency pulling associated with magnetron turn on and off. The results, displayed in Fig. 22, show that the pure magnetron signal is observed up to a power level of - 3 watts. Above 3.5 watts, however, secondary waves, centered about a frequency $8 \mathrm{MHz}$ on the low side of the carrier frequency, are detected. The low frequency spectrum also shows enhancement at this power level. From the measured power level one can compute a critical electric field $\mathrm{E}_{\mathrm{x}}$ inside the plasma of $9.4 \mathrm{~V} / \mathrm{cm}$, somewhat higher than the theoretical value of $6 \mathrm{~V} / \mathrm{cm}$. The calculation is sensitive to the assumption of the effective cross sectional area through which the power is transmitted. A radial extent of $2.5 \mathrm{~cm}$ was measured with the probe, but the height of the cross section was assumed to be the same as the long dimension of the waveguide $(5.8 \mathrm{~cm})$ :

Beyond the threshold power, it is noteworthy that the spectral interval between the carrier signal $f_{0}$ and $f_{0}-8 \mathrm{MHz}$ fills in, but the secondary signal is always about 40 db down from the carrier signal. One estimates that the total energy into the secondary waves is always less than 1\% of the energy in the carrier, even if the power level is greater than 500 times the threshold. In this experiment, then, the nonlinear aspects never dominate. It. is well to point out, however, that 
nonlinear interactions may be more important in hot, fully ionized plasmas, where collisions are unimportant, especially if there exists a large amplitude standing wave between the waveguide injector and a reflecting region within the plasma. 


\section{APPENDIX A:}

The dispersion relation for cold plasma waves is

$$
\text { where } \begin{aligned}
& a n_{\perp}^{4}-b n^{2}+c=0, \\
& a=s \\
& b=(S+P)\left(S-n_{n}{ }^{2}\right)-D^{2} \\
& c=P\left[\left(S-n_{n}^{2}\right)^{2}-D^{2}\right] .
\end{aligned}
$$

and S, P, and D are components of the plasma dielectric tensor in the notation of Stix [2]. The topology of the dispersion relation depends upon $n_{"}$ and $\Omega^{2}=\omega^{2} / \omega_{c e} \omega_{c i}$ and is shown in Fig. 25 . The regions (b) and (c) are bounded by the curve

$$
\Omega^{2}=n_{n}^{2} /\left(n_{n}^{2}+1\right)
$$

and regions (c) and (d) by the accessibility condition which can be written

$$
\Omega^{2}=n_{n}^{2} /\left(n_{n}{ }^{2}-1\right)
$$

For conditions in the parameter range (a), (b), and (c) the slow wave propagates when the density is greater than the critical density $\left(n_{c}\right)$. The maximum density to which the wave cain prupagate is determined by the equation

$$
(P-S)\left(S-n_{n}^{2}\right)-D^{2}=2 D n_{n}(1-P)^{1 / 2},
$$

which, if $\omega>>\omega_{\mathrm{ci}}$, $\omega_{\mathrm{pi}}$ reduces to

$$
\frac{n_{1}}{n_{c}}=\left[\frac{\left(n_{n}{ }^{2}-1\right)^{(1)} c e}{2 n_{1} w}\right]^{2}+1 .
$$

In region (d) the wave is said to be accessible to the 
lower hybrid layer since, in the cold plasma approximation, the slow wave can propagate to the resonance point.

In region (e) of parameter space, defined by $n_{n}<1$; there are three possible topologies for the dispersion relation. One is shown in Fig. $23\left(\right.$ e) where $n_{\|}=0$. The others differ only at densities near and greater than the lower hybrid density.

The polarization of the wave can be readily obtained from the expressions

$$
\frac{i E_{y}}{E y}=\frac{n_{n}{ }^{2}-S}{D} \quad, \frac{E_{z}}{E_{x}}=-\frac{-n_{\perp} n_{\|}}{\left(P-n_{n}{ }^{2}\right)}
$$

where $E_{z}$ is along the steady magnetic field, $E_{x}$ is along the density gradient and $E_{y}$ is perpendicular to $E_{z}$ and $E_{x}$. Some results for the condition of the experiment are shown in Fig. 24 . When $\mathrm{n}_{n}{ }^{2}>1$, just above the criticaldensity the telectric field of the slow wave is essentially along the static llayiletic ficld but well beyond the critical layer the component along the density gradient dominates. For large values of $\mathrm{n}_{\text {" }}$ the wave is almost longitudinal at densities well above the critical density. 
APPENDIX B.

The behaviour of the double waveguide radiating into a vacuum can be described most simply in terms of partial waves. The output $v_{i}$ from each arm of the $90^{\circ}$ hybrid junction can be calculated using the hybrid scattering matrix and the input vector $\left[I_{i}\right]$ representing the input signals at each arm. The output vector (the hybrid ports are numbered in a clockwise--sense) is

$$
\left[\begin{array}{l}
v_{1} \\
V_{2} \\
V_{3} \\
v_{4}
\end{array}\right]=\frac{1}{\sqrt{2}}\left[\begin{array}{llll}
0 & j & 1 & 0 \\
j & 0 & 0 & 1 \\
1 & 0 & 0 & j \\
0 & 1 & j & 0
\end{array}\right]\left[\begin{array}{l}
I_{1} \\
I_{2} \\
I_{3} \\
I_{4}
\end{array}\right]
$$

Suppose that at the mouth of the waveguides the internal amplitude reflection coefficient is $R$ and the cross-coupled amplitude coefficient is $C$. The input vector to the hybrid junction is

$$
[I]=\left[\begin{array}{c}
1 \\
j \frac{R}{\sqrt{2}} e^{2 j\left(\phi-\frac{\pi}{2}\right)}+\frac{c}{\sqrt{2}} e^{j\left(\phi-\frac{\pi}{2}\right)} \\
j \frac{c}{\sqrt{2}} e^{j\left(\phi-\frac{\pi}{2}\right)}+\frac{R}{\sqrt{2}}
\end{array}\right],
$$

0

where $\phi$ is the phase difference between adjacent guides. The inputs at arms 2 and 3 represent wave amplitude that has been reflected and cross coupled back from the outputs of arms 2 and 3 by the double waveguide. There is no input to arm 4 because 
that arm is terminated.

The power reflection coefficients measured at arms one and four are thus

$$
\begin{aligned}
R_{1} & =|C+R \cos \phi|^{2} \\
R_{4} & =|R|^{2} \sin ^{2} \phi
\end{aligned}
$$

and the total reflection coefficient of the coupler is

$$
\mathrm{R}_{\mathrm{T}}=\mathrm{R}_{1}+\mathrm{R}_{4}=|\mathrm{C}|^{2}+|\mathrm{R}|^{2}+\left(\mathrm{RC}^{\star}+\mathrm{CR}{ }^{\star}\right) \operatorname{Cos} \dot{\phi} .
$$

The cquations predict that the power reflection in the terminated fourth arm of the hybrid varies with $\phi$ with a perind of $\pi$ whereas the total reflection coefficient has a periodicy of $2 \%$. This has been observed experimentally. The experimentally determined total reflection coefficients for the double waveguide radiating into a vacuum are included in Fig. 9. We obtain

$$
\begin{aligned}
& |R| \sim 0.45 \\
& |C| \sim 0.28
\end{aligned}
$$

and $R$ and $C$ are $180^{\circ}$ out of phase. The two return waves interfere distructively when $\phi=0$ and constructively when $\phi:=180^{\circ}$ yielding the reflection versus $\psi$ shown in Fig. n. The result.s are in agreement with other measurement obtained by placing directional couplers at the second and third arms of the hybrid junction. 


\section{ACKNOWLEDGMENTS}

We gratefully acknowledge the contributions of $\mathrm{P}$. Colestock and M. Brennan in the early states of this work, and the technical assistance of J. Frangipani.

This work was supported by the United States Energy Research and Development Administration Contract E. (11-1)-3073. 


\section{REFERENCES}

[1] BRAMBILLA, M., Nucl. Fus. 16 (1976) 47 .

[2] STIX, T. H., The Theory of Plasma Waves, (McGraw-Hill, New York, 1962).

[3] GOLANT, V. E., Zh. Tekh. Fiz. 41 (1971) 2492 [Sov. Phys. Tech. Phys. - JETP 16 (1972) 1980].

[4] PARKER, R. R., M.I.T. QPR No. 102 (1971) 97.

[5] BRIGGS, R. J., PARKER, R. R., Phys. Rev. Lett. $\underline{29}$ (1972) 852 .

[6] BELLAN, P., PORKOLAB, M. , Phys. Fluids 17 (1974) 1592.

[7] TROYON, R., PERKINS, F. W., presented at Second Topical Conference on RF Plasma Heating, (Lubbock, Texas, 1974).

[8] LALLIA, P., presented at Second Topical Conference on RF Plasma Heating (Lubbock, Texas, 1974).

[9] BERS, A., Private Communicatiul.

[10] MOTLEY, R. W. , BERNABEI, S., HOOKE. W. M., JASSBY, D. L., J. Appl. Phys. 46 (1975) 3286 .

[11] BERNABEI, S., HEALD, M. A., HOOKE. W. M., PAOLONI, F. J., Phys. Rev. Lett. 34 (1975) 866 .

[12]. HOOKE, W. M., BERNABEI, S., Phys. Rev. Lett. 28 (1972) 407.

[13] Colestock, P. L., GeTTY, W. D., presented at second Topical Conference on RF Plasma Heating, (Lubbock, Texas, 1974).

[14] PORKOLAB, M., ARUNASALAM, V., LUHMANN, JR., N. C., Plasma Phys: 17 (1975) 405 .

[15] PORKOLAB, M., Symposium on Plasma Heating in Toroidal Devices (Proc. Symp. Varenna, 1974) Editrice Compositori, Bologna, Italy, (1974). 
[16] CHU, T. K., BeRnabeI, S., MOtLEy, R. W., Phys. Rev. Lett. 31 (1973) 211 .

[17] HOOKE, W. M., BERNABEI, S., Phys. Rev. Lett. 29 (1972) 1218.

[18] MORAlEs, G. J., LEE, Y. C., Phys. Rev. Lett. 14 (1975) 930. 


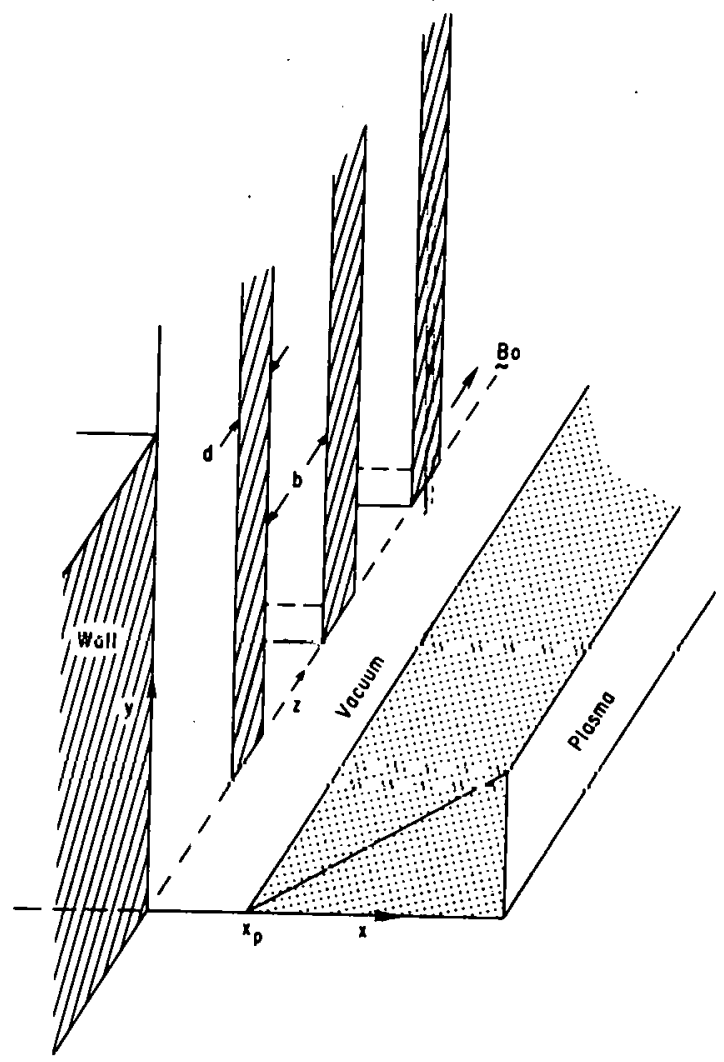

763656

Fig. 1. Model of the waveguide array and plasma. The individual waveguide width is $b$, the wall thickness is $d^{-}$ and the plasma edge is at a distance $x_{p}$ from the waveguides.

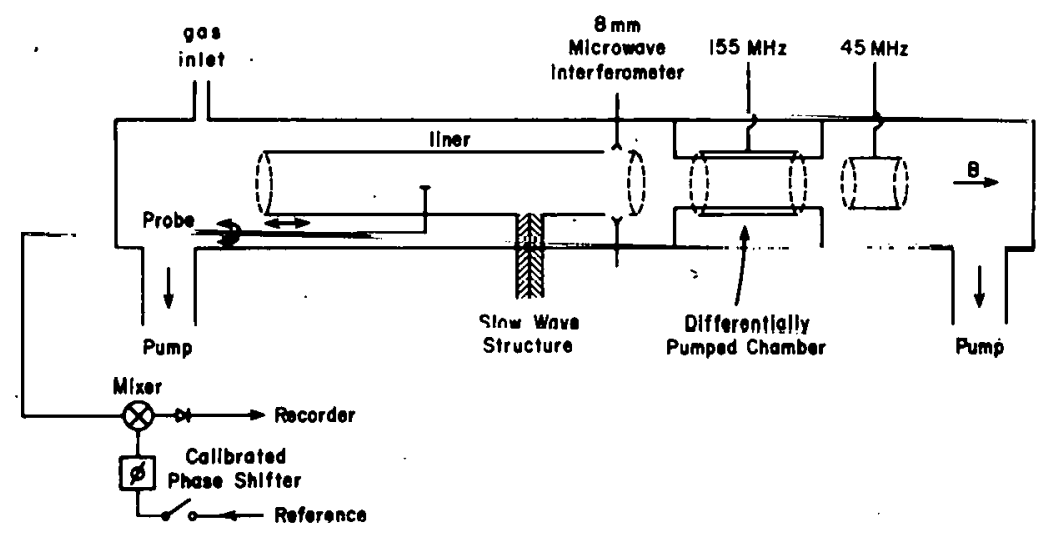

763825

Fig. 2. Schematic diagram: of the experimental apparatus. 


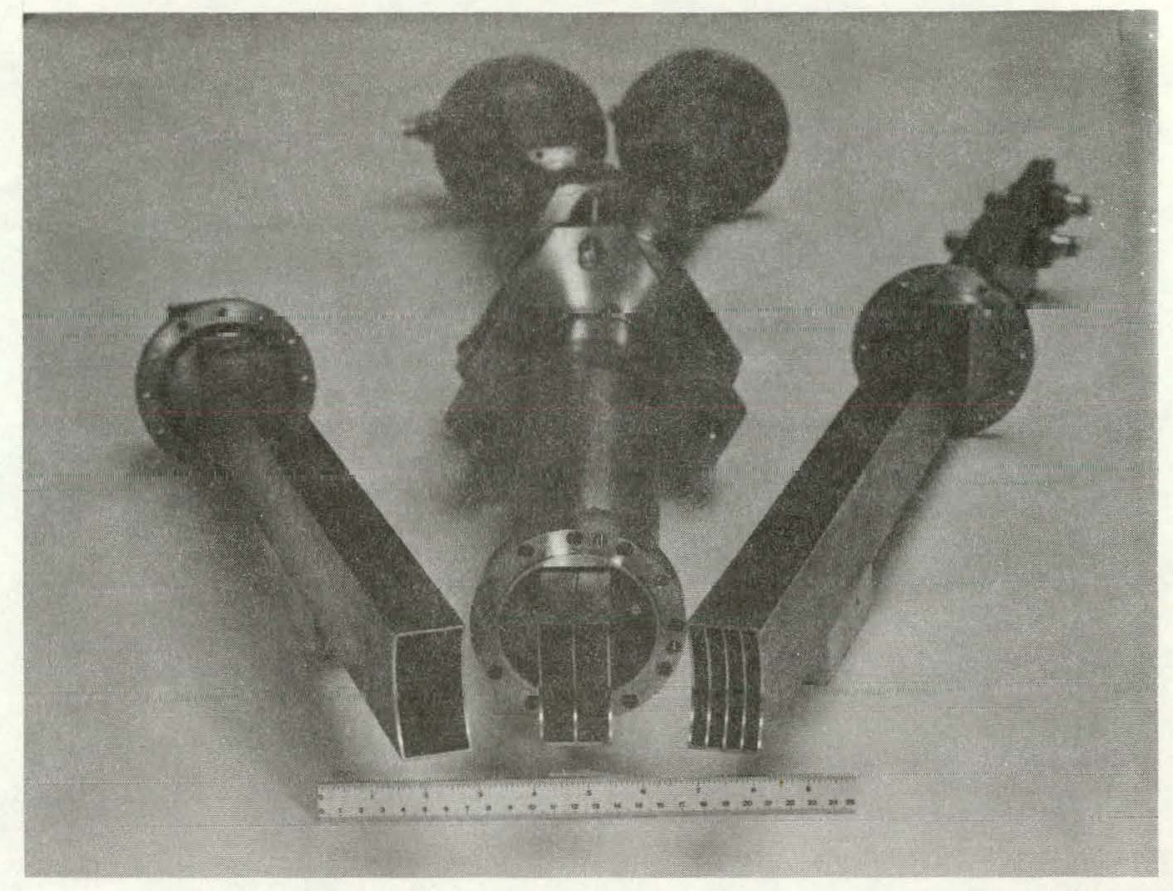

753334

Fig. 3. A photograph of the single, double, and quadruple waveguide arrays.

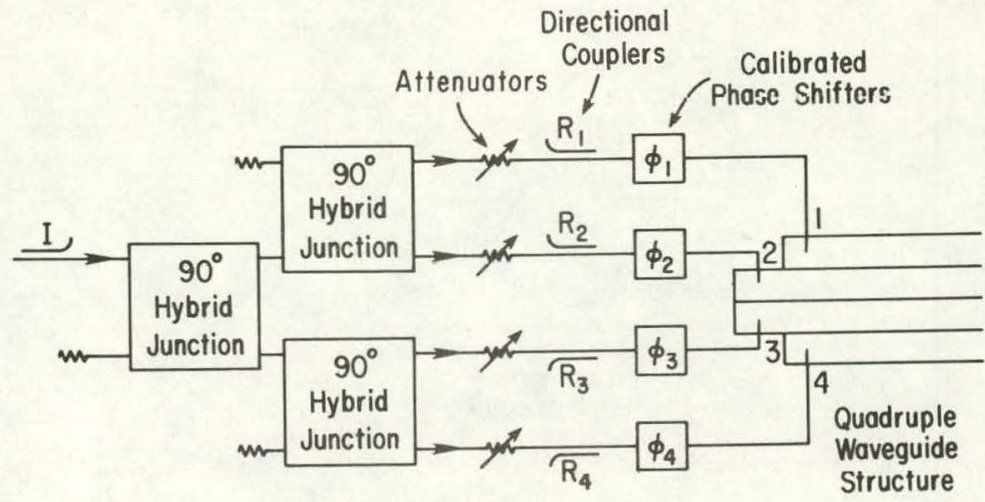

763639

Fig. 4. Schematic diagram of the external circuit which energizes the quadruple waveguide array. 


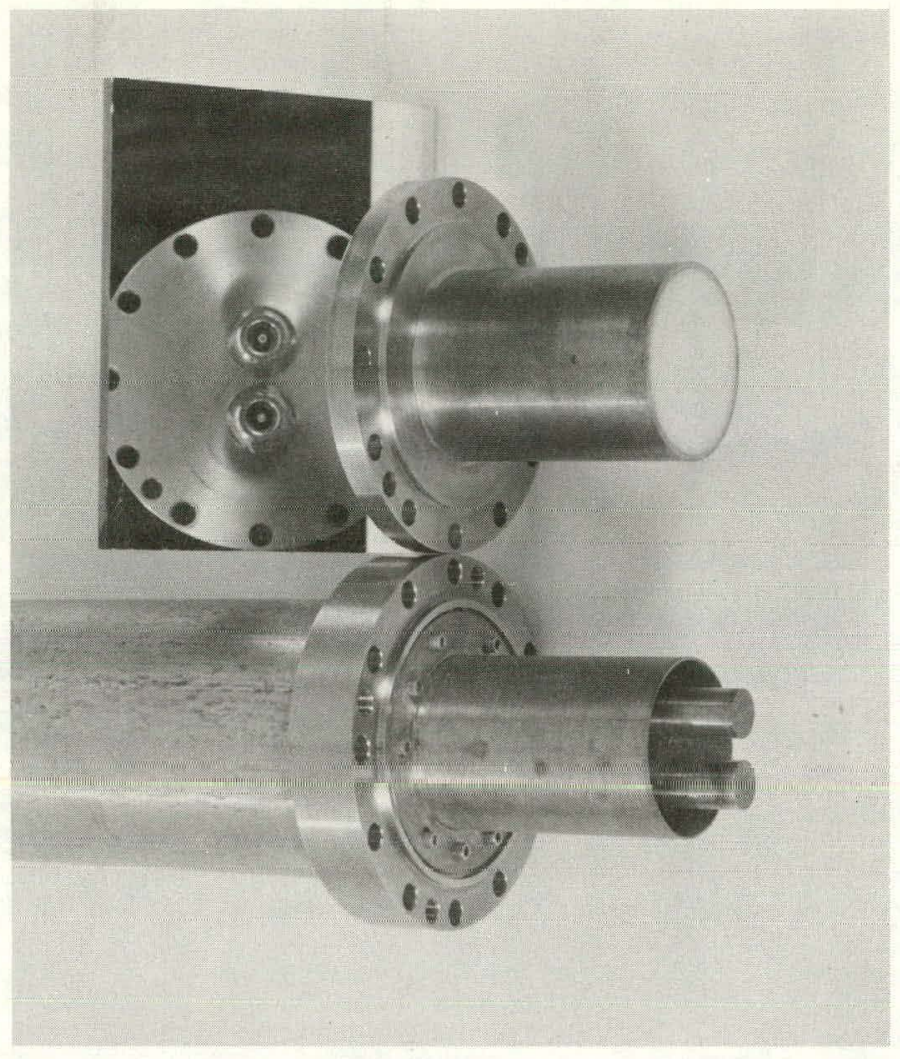

763374

Fig. 5. A photograph of the shielded pair transmission line antenna and the circular 'l'M dielectric-loaded waveguide.

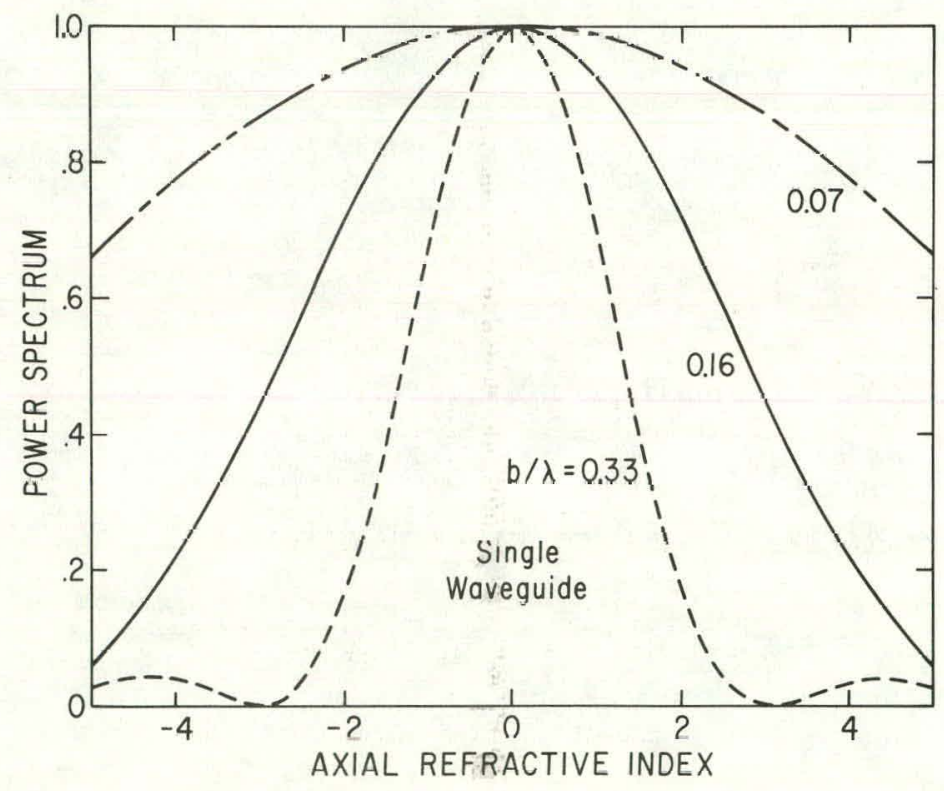

763653

Fig. 6. The nominal aperture spectra for single waveguides of various widths. 


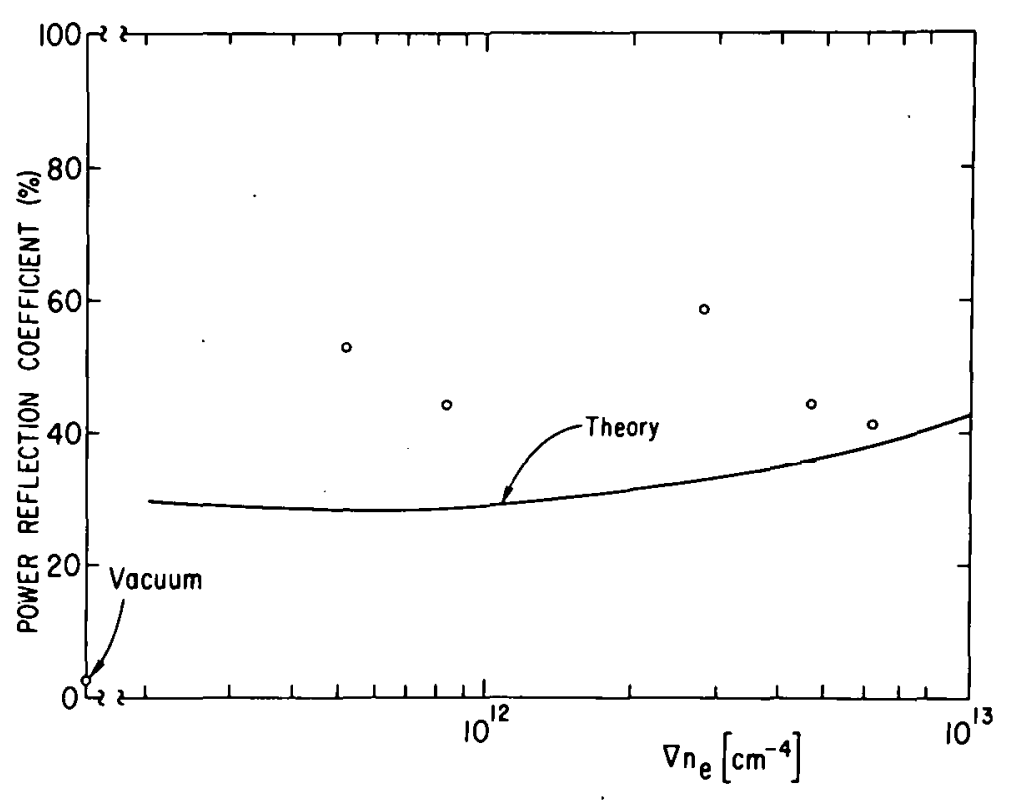

.763648

Fig. 7. The power reflection coefficient versus plasma density gradient for a single waveguide of width $2.8 \mathrm{~cm}$ positioned at the plasma edge $(b / \lambda \approx 0.32)$. The solid line represents theoretical results for the same conditions.

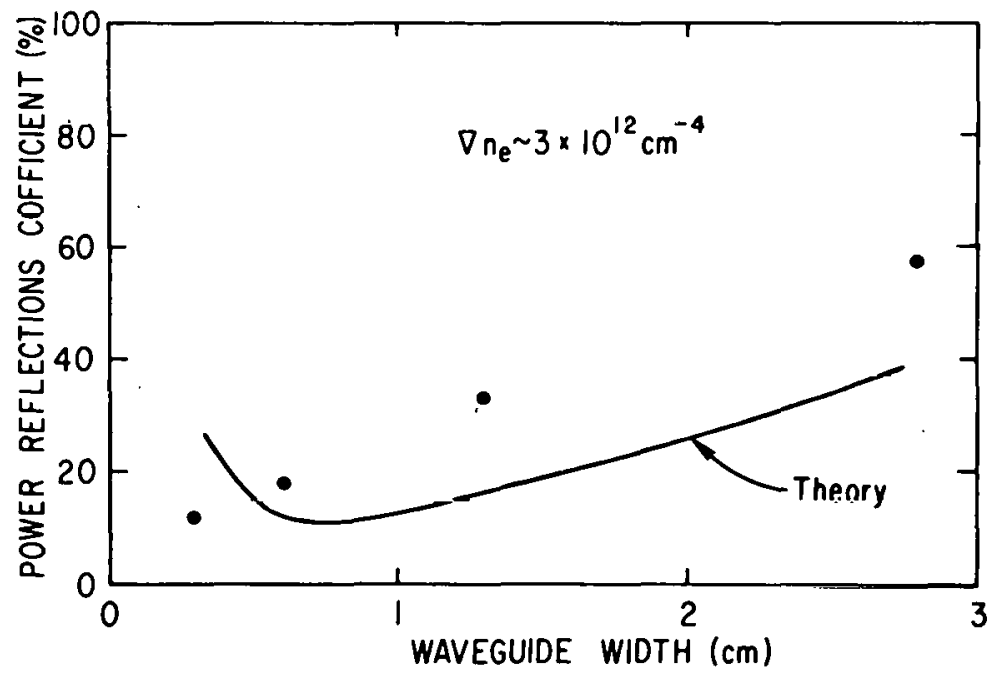

763647

Fig. 8. The power reflection coefficiegnt versus waveguide width for a density gradient of $3 \times 10^{12} \mathrm{~cm}^{-4}(\lambda=8.8 \mathrm{~cm})$ : The solid line is the theoretical result. 


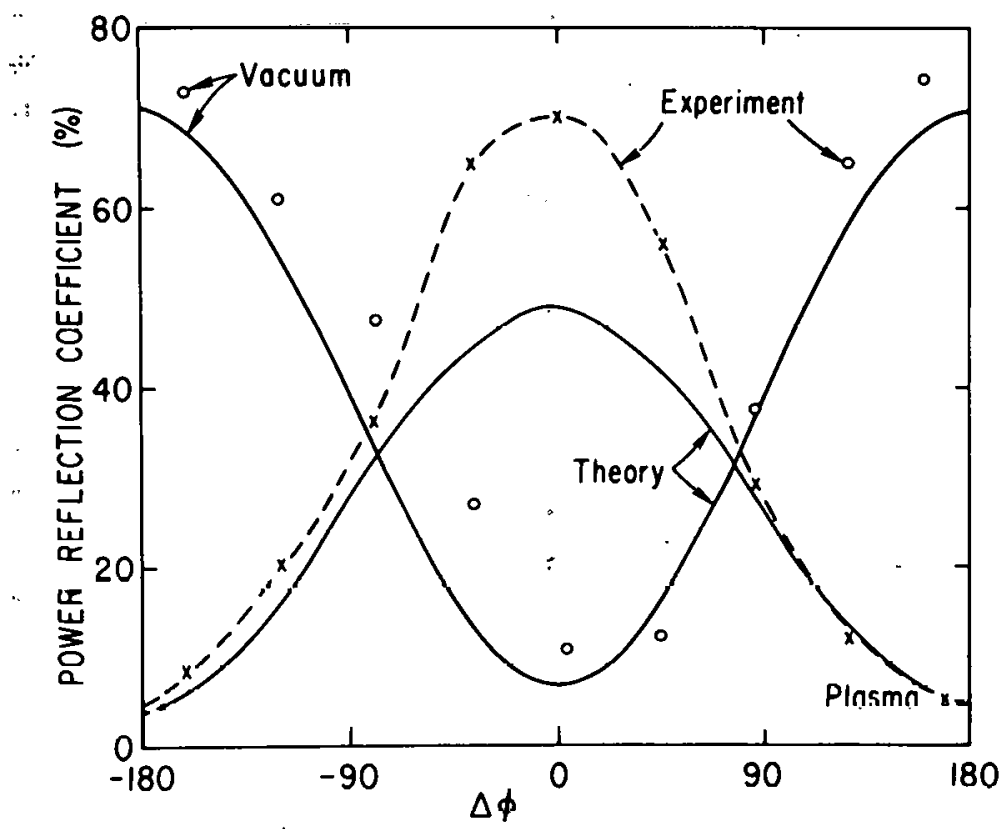

763652

Fig." 9. The theoretical and experimental power reflection coefficient versus phase difference for the double waveguide antenna. Results are for the vacuum situation and for a plasma gradient of $9 \times 10^{12} \mathrm{~cm}^{-4}$.

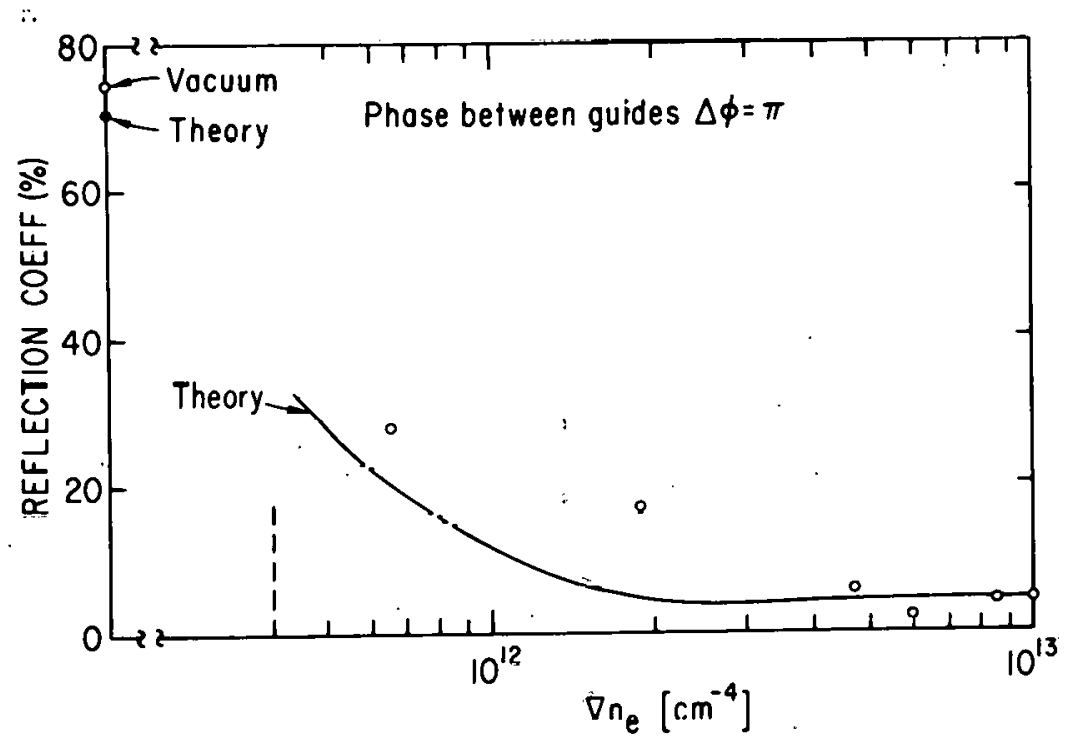

763644

Fig. 10. The power reflection coefficient versus density gradient for the double waveguide antenna when the phase difference between guides is $180^{\circ}$. The solid line is the theoretical prediction. 


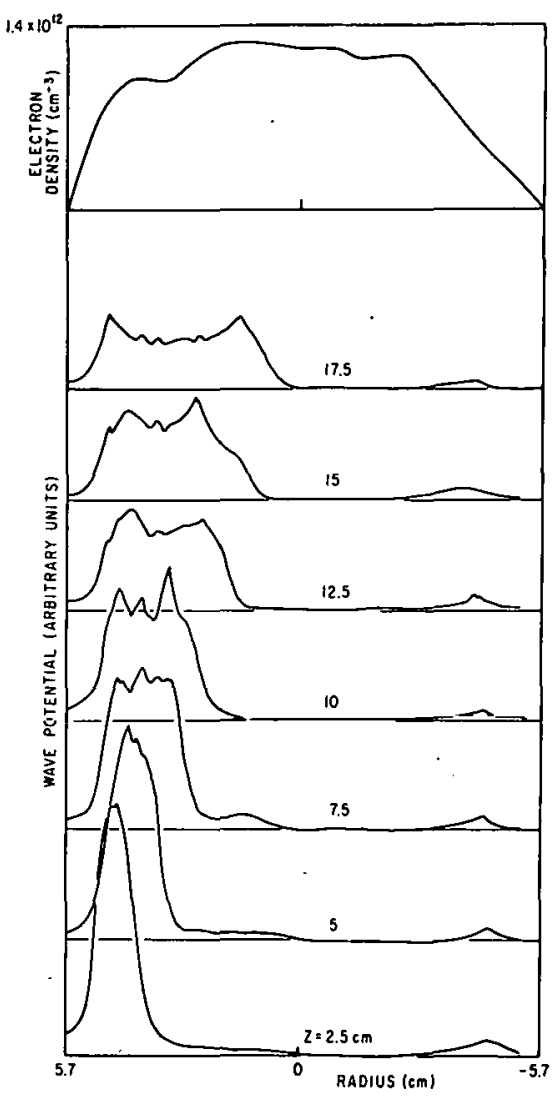

763641

Fig. 11 (a). Electron density as a function of radius, $\mathrm{n}_{\max } \simeq 10 \mathrm{n}_{\mathrm{c}}$ : (b) Observed wave magnitude as a function of radius at maxcessive planes downstream of the double waveguide antenna (at $z=0$ ). $\Delta \phi=180^{\circ}$.

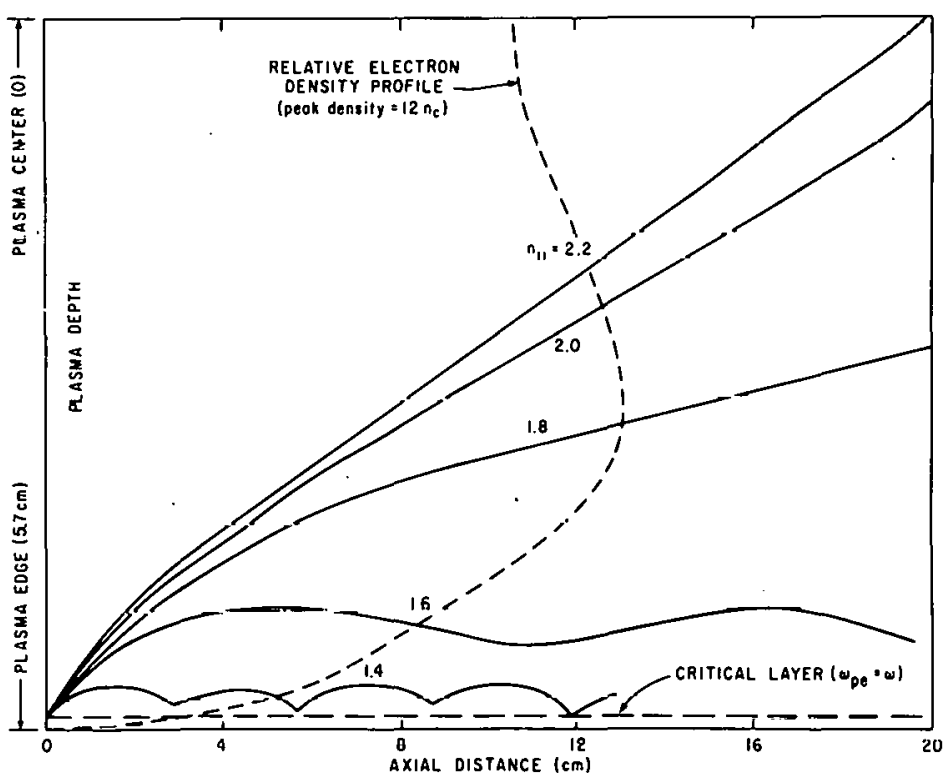

763824

Fig. 12. Calculated ray trajectories for waves of different axial wavelengths. The dashed curve is the assumed electron density distribution. 


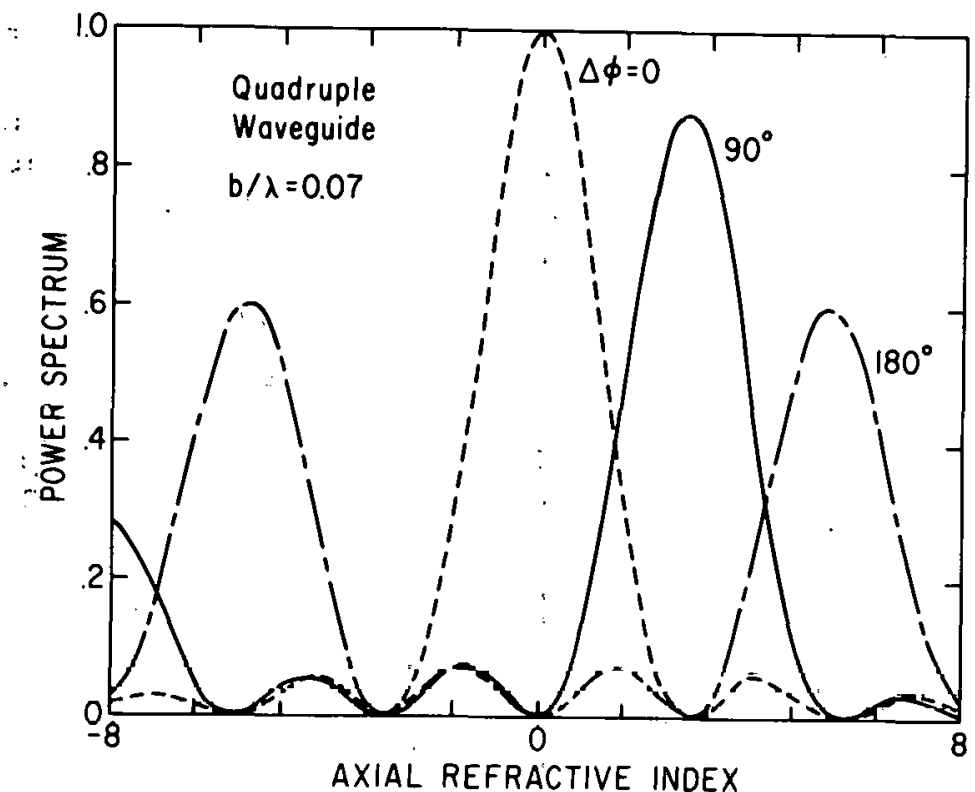

763654

Fig. 13. The nominal aperture power spectrum for the quadruple waveguide antenna when the phase difference between adjacent guides is $0,90^{\circ}$ and $180^{\circ}$. The individual waveguide width is $0.6 \mathrm{~cm}$.
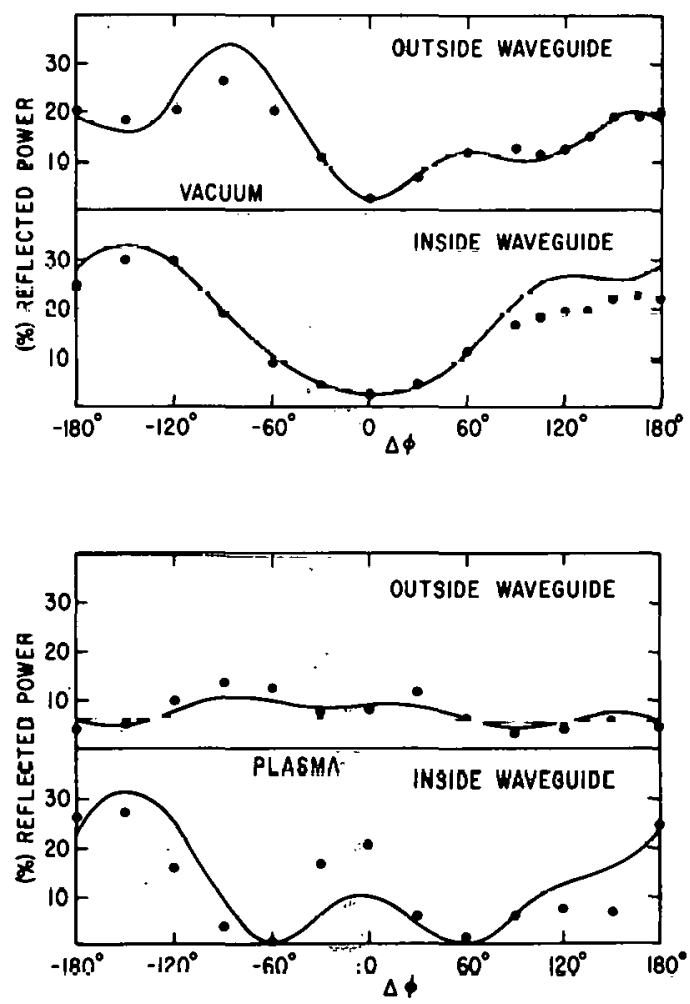

Fig. 14. (a). Power reflection coefficients versus phase difference between adjacent guides for the quadruple array radiating into a vacuum. (b) Power reflection coefficients versus phase difference for the quadruple array radiating into a plasma gradient of $5.4 \times 10^{12} \mathrm{~cm}^{-4}$. 


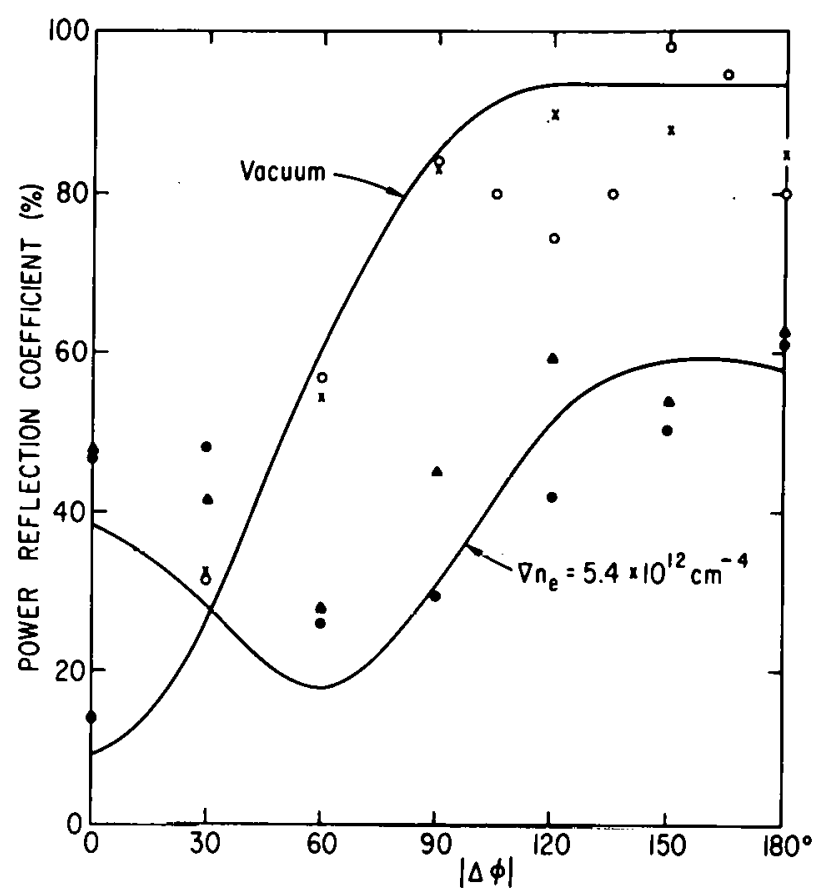

763650

Fig. 15. Total Power reflection coefficeint versus phase difference between adjacent guides of the quadruple array. The solid lines represent the theory.

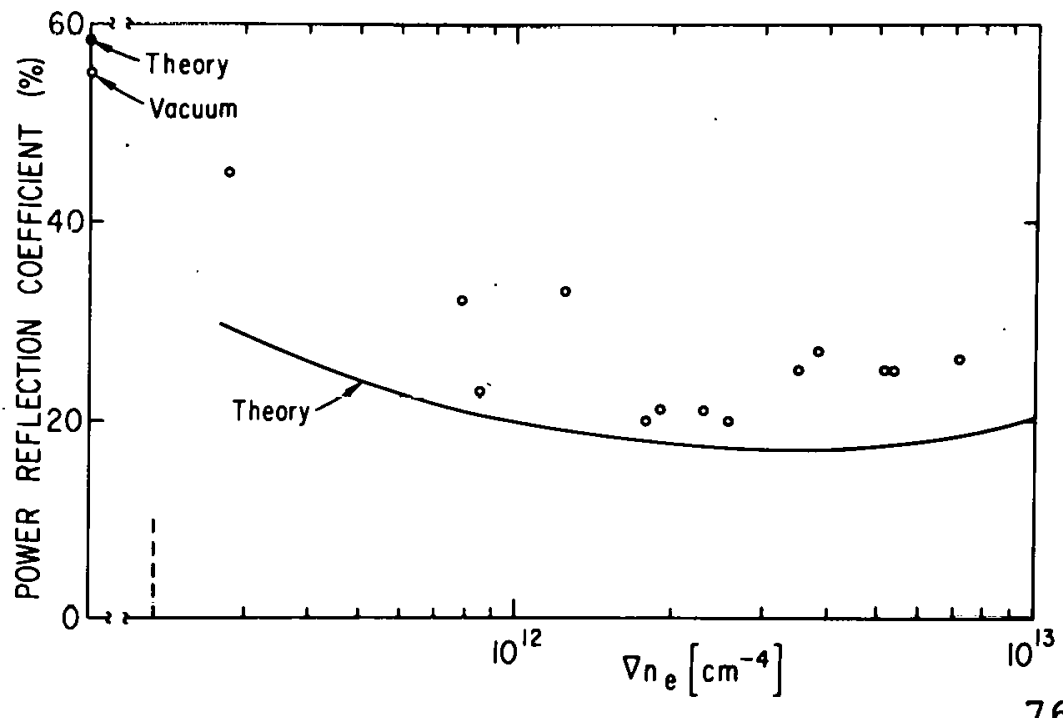

763645

Fig. 16. The experimental and theoretical results for the power reflection coefficient versus density gradient for the quadruple array with 600 phase shift between adjacent yuides. 


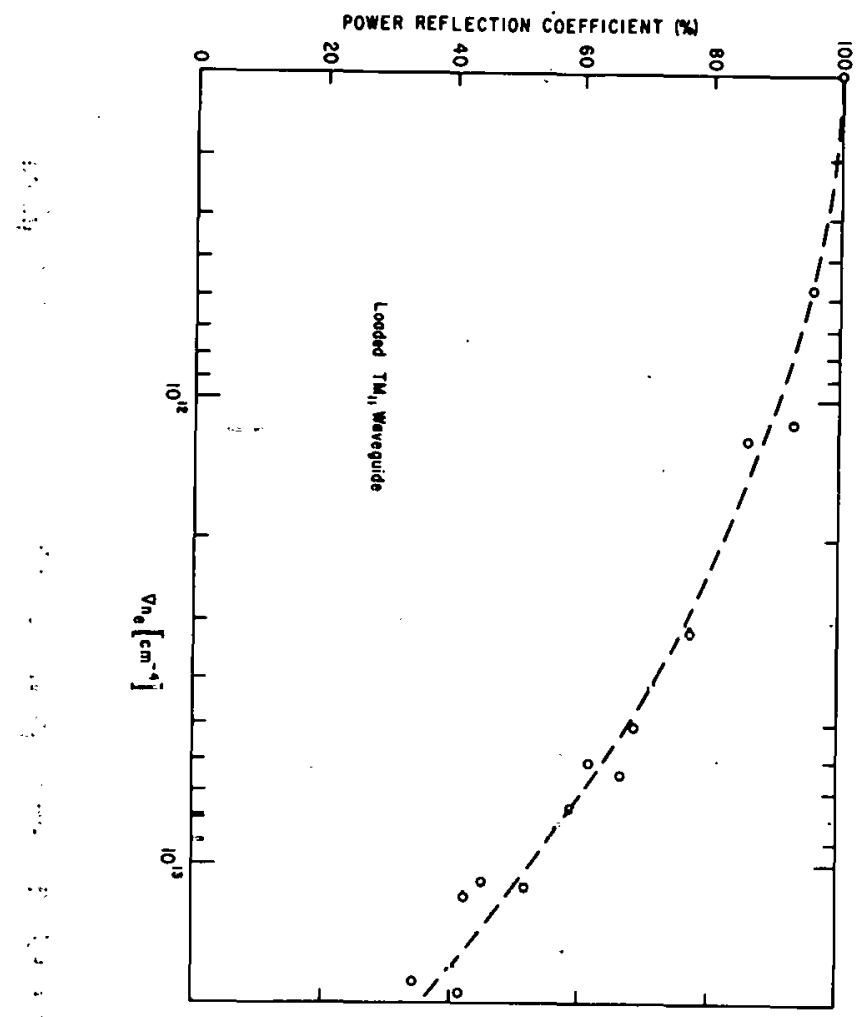

763649

Fig. 17. The experimentally measured power reflection dielectric-loaded waveguide.

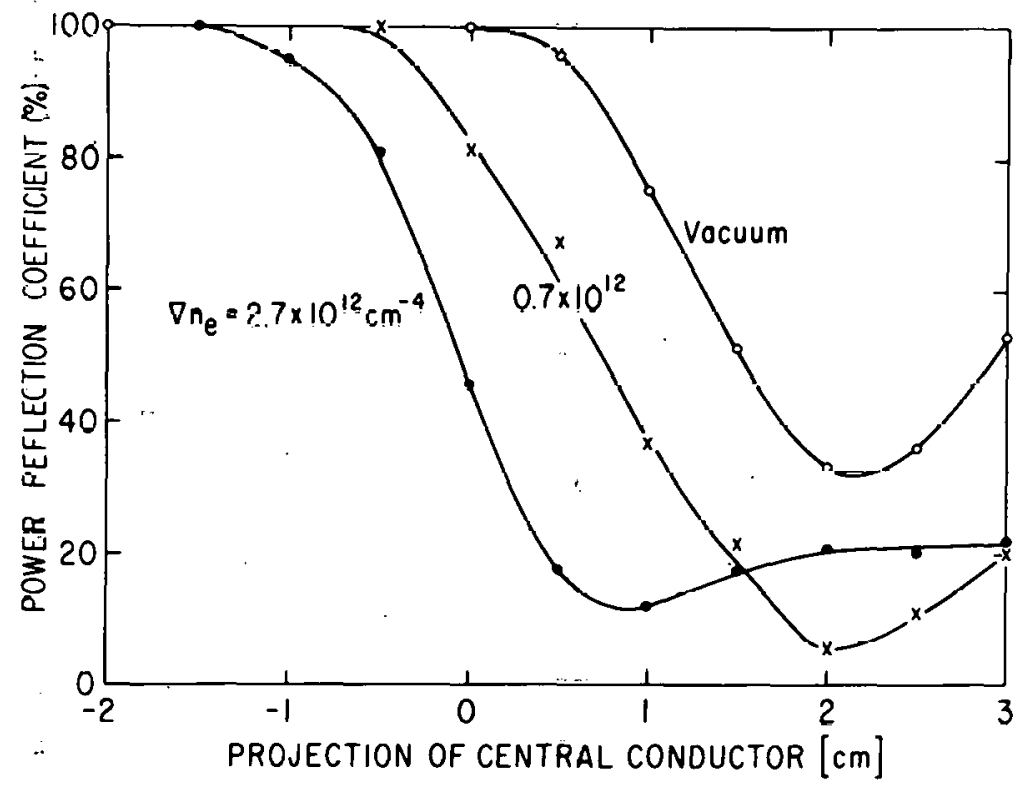

763651

Fig. 18. The experimentally measured power reflection coefficient for the shielded pair transmission line antenna when the position of the inner conductors is varied. 


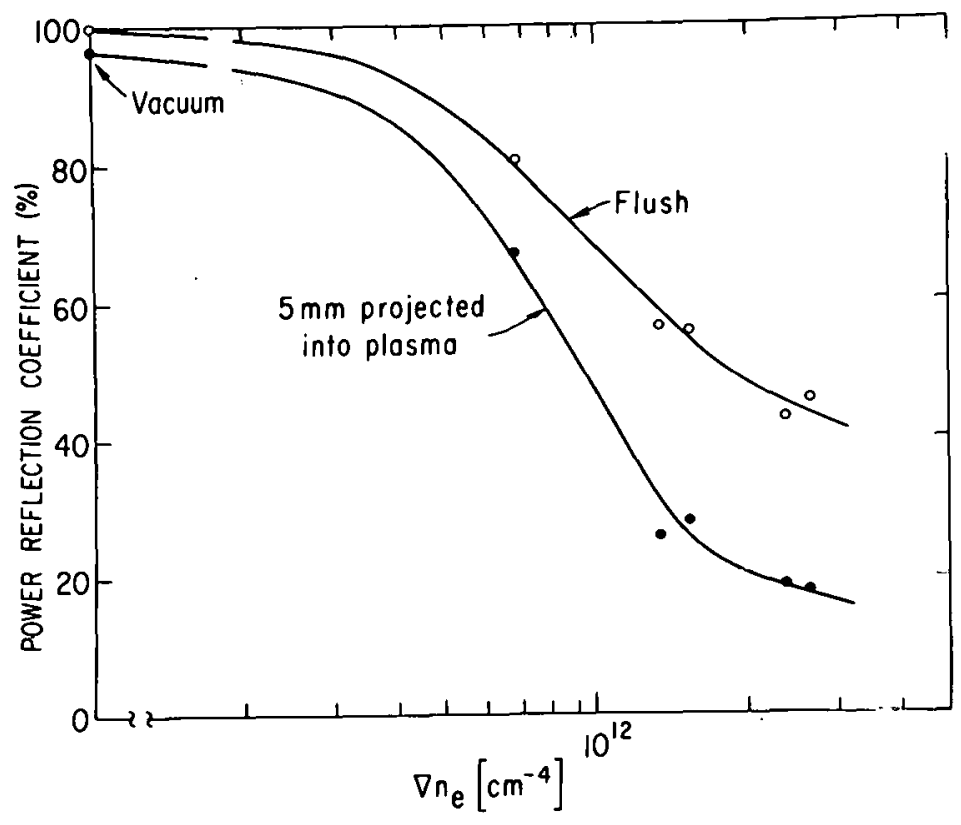

763646

Fig. 19. The measured power reflection coefficient of the coaxial transmission line antenna for various plasma gradients. Results are given for when the inner conductors are flush with outer shield and for when they are projected $5 \mathrm{~mm}$ into the plasma chamber.

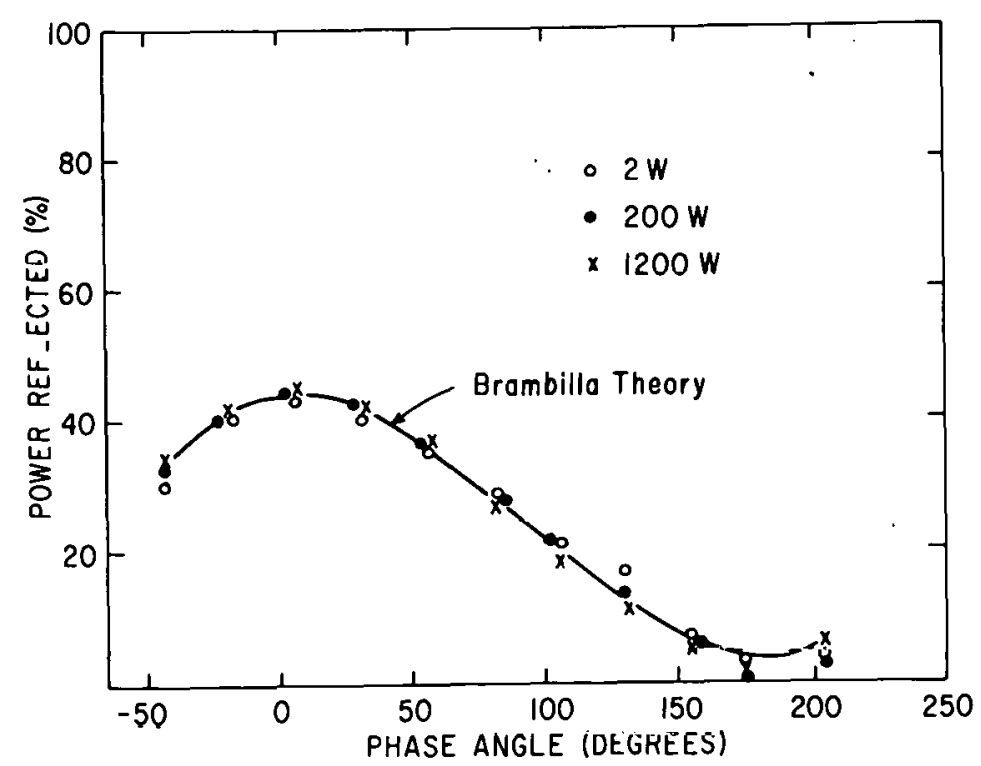

756071

Fig. 20. The power reflection coefficient versus phase difference between guides of the teflon loaded double waveguide antenna. The points represent experimental results for various input powers and the solid line is the theoretical results derived using the experimentally measured density gradient of $101 \mathrm{~cm}^{-4}$. 


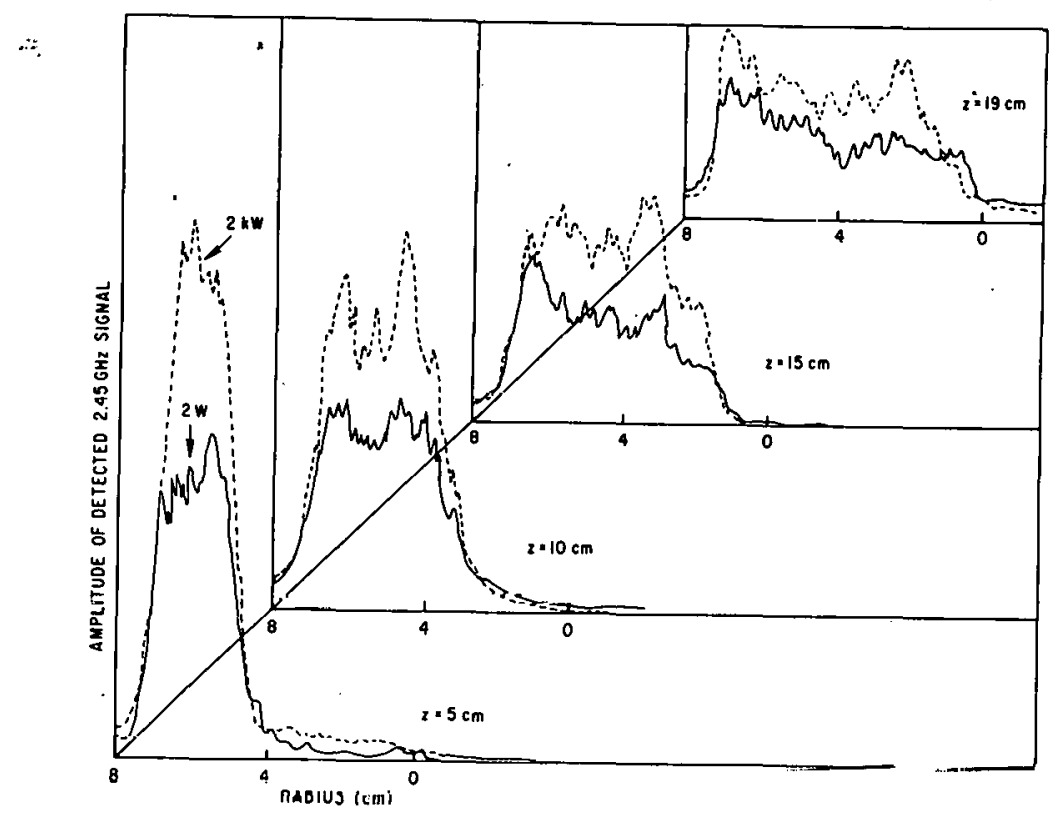

756038

Fig. 21. The wave magnitude at various positions downstream of the antenna for input powers of 2 watts (solid curves) and $2 \mathrm{~kW}$ (dashed curves).

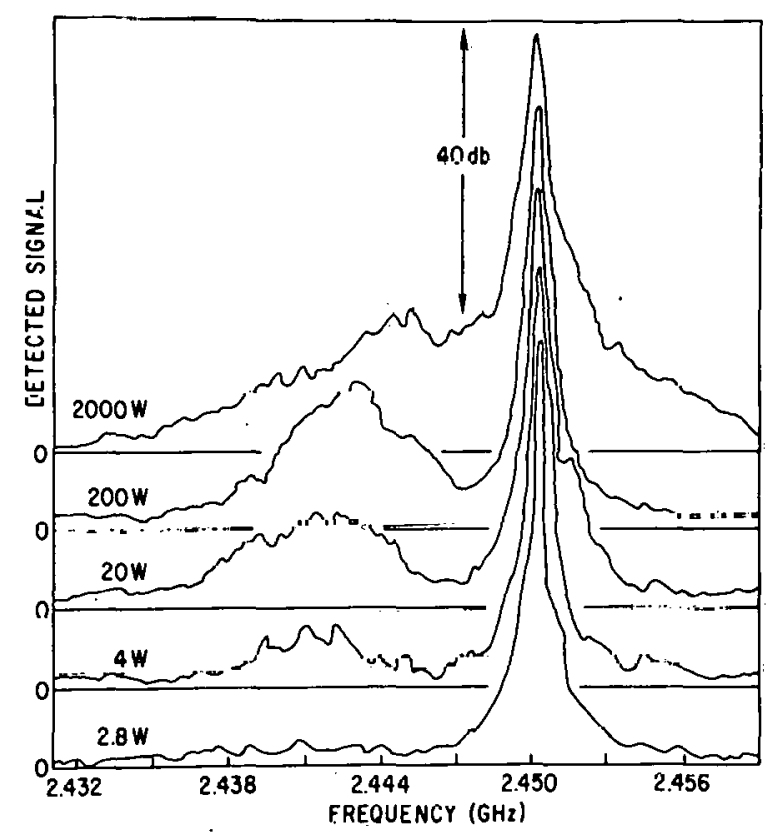

763657

Fig. 22. The time resolved wave spectra for increasing input power. 

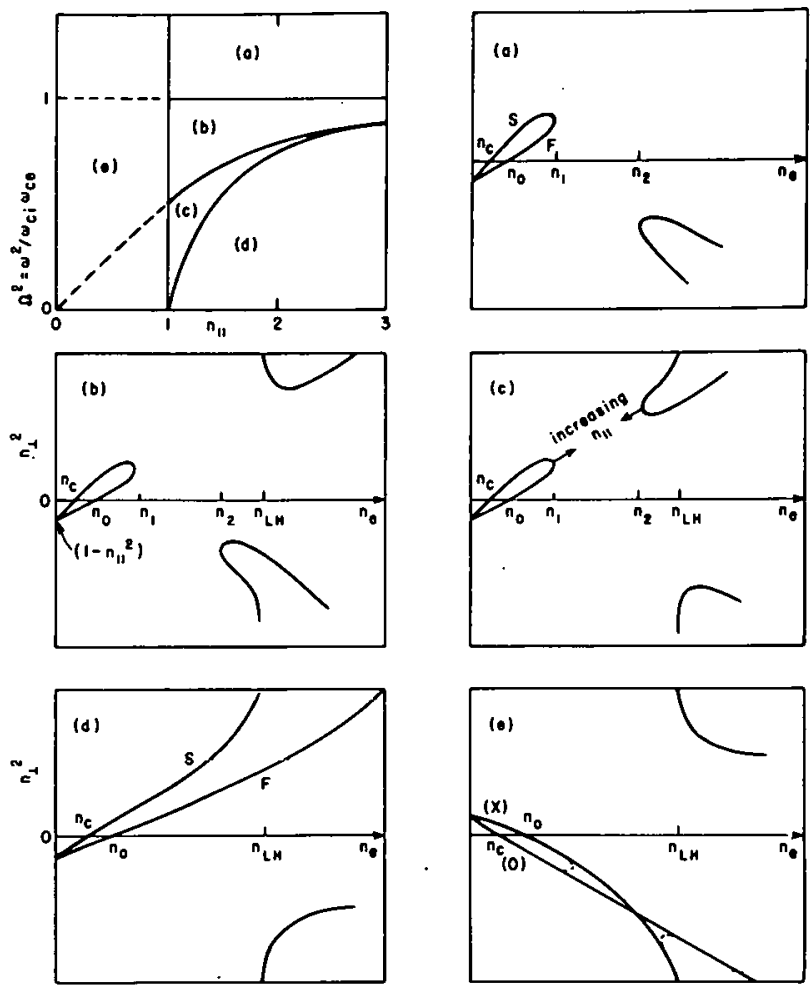

763655

Fig. 23. The topology of the dispersion relations for slow and fast plasma waves. Diagrams (a) to (e) demonstrate the perpendicular refractive index versus electron density for waves of varying axial wavelengths. The upper left hand diagram defines the regions of parameter space.

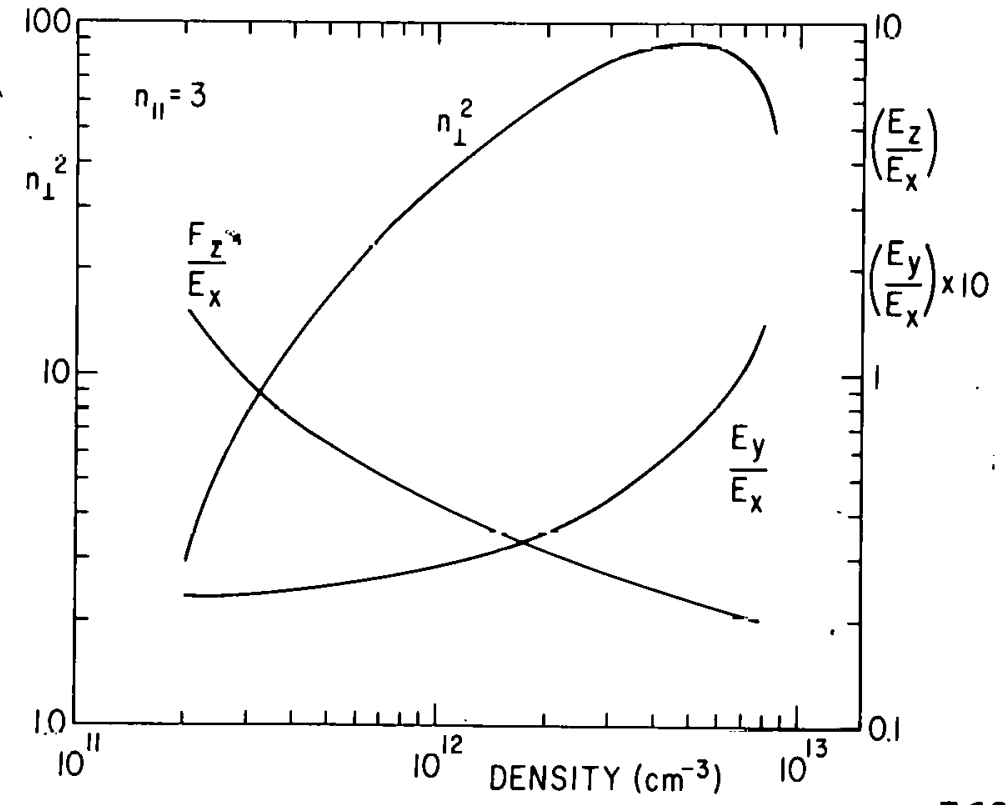

763643

Fig. 24. The relative magnitude of component electric fields for the slow wave with $n_{n}=3$ versus electron density. The perpendicular refractive index is also included. 